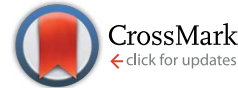

Cite this: RSC Adv., 2015, 5, 7495

Received 6th October 2014 Accepted 15th December 2014

DOI: $10.1039 / c 4 r a 11852 a$

www.rsc.org/advances

\title{
Synthesis and properties of molybdenum disulphide: from bulk to atomic layers
}

\begin{abstract}
Intek Song, ${ }^{\text {ab }}$ Chibeom Park $^{\text {ab }}$ and Hee Cheul Choi ${ }^{\star a b}$
Molybdenum disulphide $\left(\mathrm{MoS}_{2}\right)$ has been one of the most interesting materials for scientists and engineers for a long time. While its bulk form has been in use in conventional industries as an intercalation agent and a dry lubricant for many years, its two-dimensional forms have attracted growing attention in recent years for applications in nano-electronic applications. Specifically, the single layer form of $\mathrm{MoS}_{2}$ shows significant potential as a semiconductor analogue of graphene. These exciting applications are spread over many fields, from flexible and transparent transistor devices, to low-power, high efficiency biological and chemical sensing applications. This Review Article, for the first time, provides a comprehensive overview of the synthesis, structural polytypes, properties, and applications of bulk, few layer, and single layer $\mathrm{MoS}_{2}$.
\end{abstract}

\section{Introduction}

Layered crystalline materials have attracted researchers for several decades. Their unique crystallographic structure, as the term "layered" implies, is composed of separate twodimensional (2D) layers, between which the van der Waals (vdW) interaction exists in place of ionic or covalent bonding., ${ }^{1,2}$ Therefore, most of their physical properties, such as charge carrier transport or mechanical friction, are anisotropic. Properties along the in-plane direction significantly differ from the out-of-plane counterparts. ${ }^{2-4}$ Historical applications of such structural uniqueness were confined to host lattices in intercalation chemistry ${ }^{5-7}$ or dry lubricants in mechanics. ${ }^{8}$ However, recent experimental advances have found these materials are

${ }^{a}$ Center for Artificial Low Dimensional Electronic Systems, Institute for Basic Science (IBS), 77 Cheongam-Ro, Nam-Gu, Pohang, 790-784, Korea.E-mail: choihc@postech.edu ${ }^{b}$ Department of Chemistry, Pohang University of Science and Technology (POSTECH), 77 Cheongam-Ro, Nam-Gu, Pohang, 790-784, Korea capable of realising 2D nanomaterials, when the individual layers are isolated. ${ }^{-11}$ These isolated single or few layers have been good topics for the fundamental research, because some of their properties are quite distinct from bulk counterparts. Researchers also expect that they have great utility in various fields, such as flexible and transparent components in nanoelectronics, active components for chemical sensors, catalysts for various electrochemical reactions, and so forth. ${ }^{12-14}$

Among a variety of $2 \mathrm{D}$ nanomaterials, a growing number of researchers have recently focused on two-dimensional molybdenum disulphide $\left(\mathrm{MoS}_{2}\right)$, as witnessed in the exponentially rising number of publications on this subject (Fig. 1). Bulk $\mathrm{MoS}_{2}$, like graphite, is a layered material, where each layer of $\mathrm{MoS}_{2}$ is composed of three-atom-thick S-Mo-S stacks (Fig. 2a). Each layer of $\mathrm{MoS}_{2}$ is often referred as a "single layer" or a "monolayer" by convention. This is contrary to other chalcogenides such as $\mathrm{Bi}_{2} \mathrm{Se}_{3}$, in which case it is rather called a "quintuple layer" $(\mathrm{QL})$, reflecting the atomic structure of $1 \mathrm{QL}$, i.e. a stack of Se-Bi-Se-Bi-Se. Such convention, however, rarely applies to $\mathrm{MoS}_{2}$ and other transition

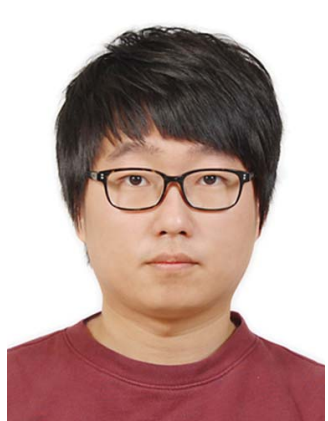

Intek Song is a Ph. D. student in the Department of Chemistry at POSTECH, under the guidance of Prof. H. C. Choi. He obtained his B.S. degree in the Department of Material Science and Engineering, POSTECH in 2012. His current research topic is the development of novel methods to grow various $2 D$ nanomaterials in large scale. He receives a Global Ph.D. Fellowship grant from Ministry of Education, Korea.

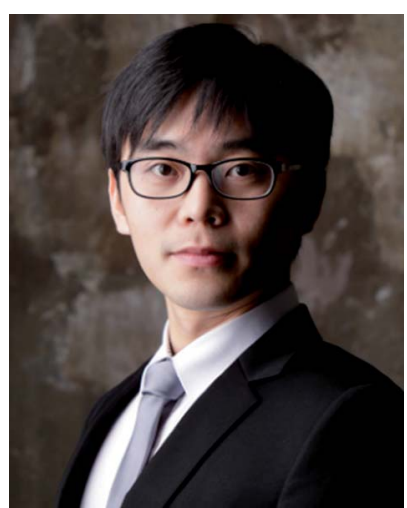

Chibeom Park received his B.S. in Material Science and Engineering at POSTECH in 2008 and his Ph.D. in the Department of Chemistry at POSTECH. During his Ph.D course, he spent 1 year at Cornell University as a visiting student. He is currently a postdoctoral fellow at POSTECH studying the electrical properties of chemically doped carbon-based materials under the guidance of Prof. H. C. Choi. 


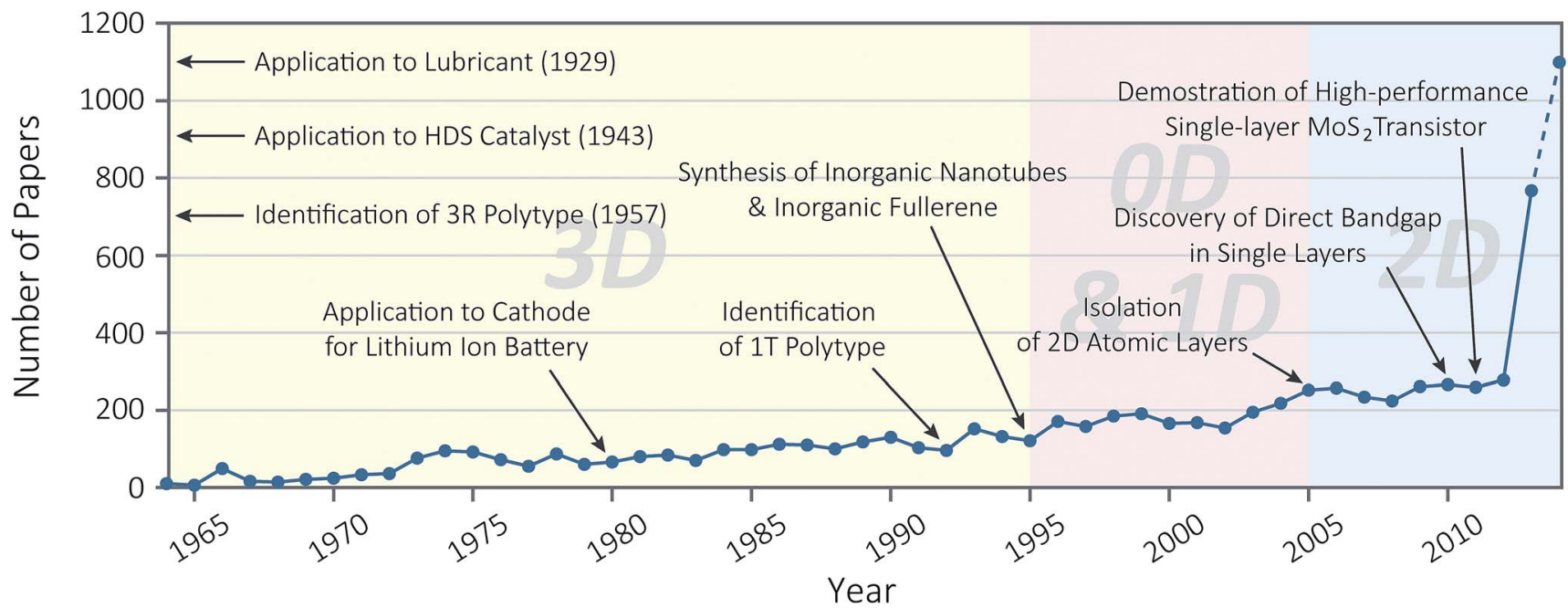

Fig. 1 History of molybdenum disulphide research for last 50 years (1964-2014). The graph shows the annual number of journal publications, of which title, abstract or keywords contains word "molybdenum disulphide", "molybdenum disulfide", or "MoS 2 ", based on Scopus ${ }^{\circledR}$ query. Note that the number in Year 2014 (1099) is extrapolated from the number of publications till 30 September, 2014 (824). Major achievements are denoted in the graph, based on the following references: "Application to Lubricant (1929)" ref. 18; "Application to HDS Catalyst (1943)" ref. 19; "Identification of 3R Polytype (1957)" ref. 62; "Application to Cathode for Lithium Ion Battery" ref. 20; "Identification of 1T Polytype" ref. 37; "Synthesis of Inorganic Nanotubes \& Inorganic Fullerene" ref. 21; "Isolation of 2D Atomic Layers" ref. 10, "Discovery of Direct Bandgap in Single Layers" ref. 25, "Demonstration of High-performance Single-layer MoS 2 Transistor" ref. 26.

metal dichalcogenides, such as a "triple layer," although singlelayer $\mathrm{MoS}_{2}$ or monolayer $\mathrm{MoS}_{2}$ is in fact three atoms thick. In addition, depending on the coordination of Mo atoms within a single layer and the stacking order of single layers, there are three known polytypes-namely $1 \mathrm{~T}, 2 \mathrm{H}$, and $3 \mathrm{R}-\mathrm{of} \mathrm{MoS}_{2}$.

$\mathrm{MoS}_{2}$, especially of naturally abundant $2 \mathrm{H}$ polytype, has a long history of study and applications, because of its abundance in the Earth's crust. ${ }^{15-17}$ Its major industrial applications include dry lubricants, ${ }^{18}$ hydrodesulphurisation catalysts, ${ }^{19}$ and cathode materials for lithium ion batteries. ${ }^{20}$ Also, $\mathrm{MoS}_{2}$ nanotubes and fullerenes are one of the first inorganic analogues of carbon nanotubes and fullerenes to be synthesised, but their proposed applications are still restricted to traditional lubrication. ${ }^{21-24}$ However, the discovery of direct bandgap of $1.9 \mathrm{eV}$ from singlelayer $\mathrm{MoS}_{2}$ has opened up new possibilities of utilising $\mathrm{MoS}_{2}$ in

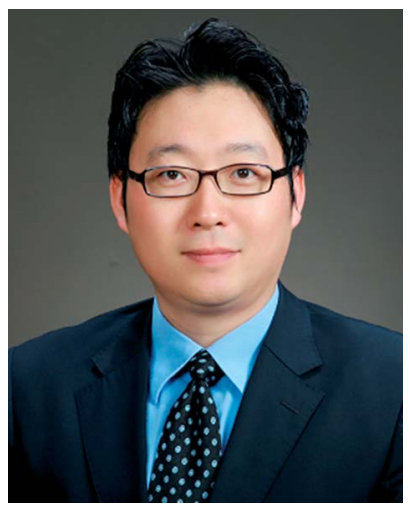

Hee Cheul Choi is a Hongdeok Young Chaired Professor in the Department of Chemistry at POSTECH and Group Leader of CALDES of IBS. He obtained his Ph.D. in Chemistry from Purdue University (2001), then spent 2 years as a postdoctoral research associate at Stanford Chemistry. His work has encompassed many aspects of materials chemistry including synthesis, surface chemistry, device fabrication, and characterization of carbon-based low dimensional and quantum scale structures. electronics. ${ }^{10,13,14,25,26}$ This analogous form of graphene, a wellknown robust semi-metal, completes the palette of $2 \mathrm{D}$ materials, spanning all of conductor (graphene), insulator (hexagonal boron nitride), and now semiconductor (single-layer $\mathrm{MoS}_{2}$ ). Furthermore, the discovery of the valley polarisation in single layers offers a novel, prospective carrier of information..$^{27-30}$ Despite its indirect bandgap, two to several layers of $\mathrm{MoS}_{2}$, or "few-layer $\mathrm{MoS}_{2}$ ", still have attractive properties for various electronic devices because of its semiconductivity. Various studies on single- to few-layer $\mathrm{MoS}_{2}$ have ensured its suitability for flexible, transparent, low-power devices in electronics. ${ }^{26}$ Finally, it can be utilised in important chemical applications such as hydrogen evolution reaction ${ }^{31,32}$ and chemical sensing. ${ }^{33}$

Attracted by these immense potentials, an increasing number of researchers turn their attention to explore this material. Despite such increasing popularity, there is no comprehensive review, to the best of our knowledge, concentrating on atomic layers of $\mathrm{MoS}_{2}$. Moreover most of the previous review papers on $\mathrm{MoS}_{2}$ mainly focuses on the most abundant $2 \mathrm{H}$ polytype only, although the $3 \mathrm{R}$ and $1 \mathrm{~T}$ types have their own interesting properties. Herein, we review, for the first time, synthesis, properties and applications of $2 \mathrm{D}$ atomic layers of $\mathrm{MoS}_{2}$, including a concise overview of its bulk form and of its various polytypes. This review aims to provide a brief introduction of this attractive material to readers from various backgrounds in order to help them to understand and utilise this nanomaterial for their own demands. To this end, this review article is composed of following contents. The first part (Section 2) is a prelude to 2D layers, where we briefly summarise the fundamental nature and the historical application of various polytypes of bulk $\mathrm{MoS}_{2}$. The second part (Section 3) provides an up-to-date review on the studies regarding the 
a

C
$1 T$

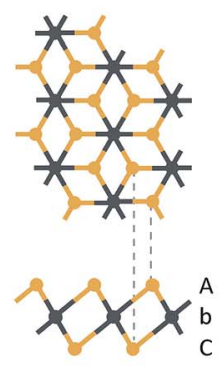

$2 \mathrm{H}$

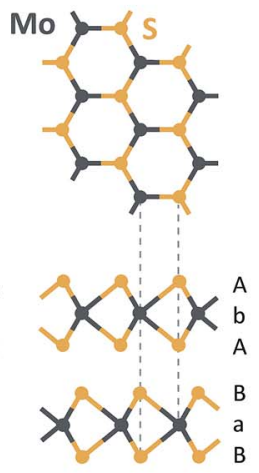

$3 R$

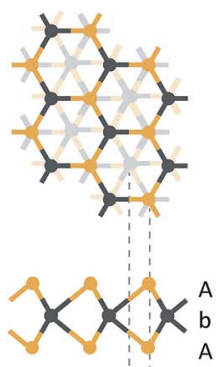

Lateral View of

Single Repeating Unit

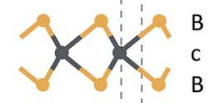

$\mathrm{Ki}^{{ }^{\mathrm{a}}}$ b

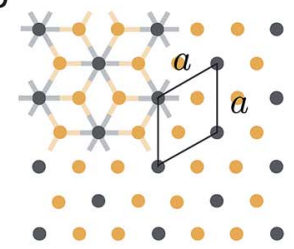

Undistorted

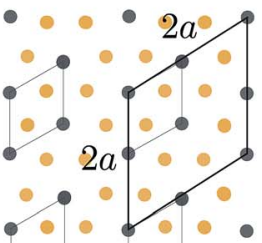

$2 a \times 2 a$

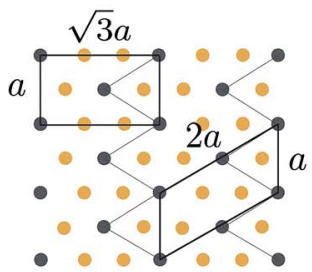

$2 a \times a$ or $\sqrt{3} a \times a$

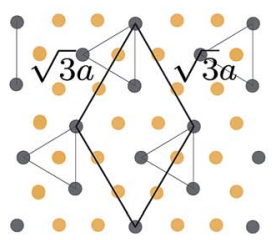

$\sqrt{3} a \times \sqrt{3} a$

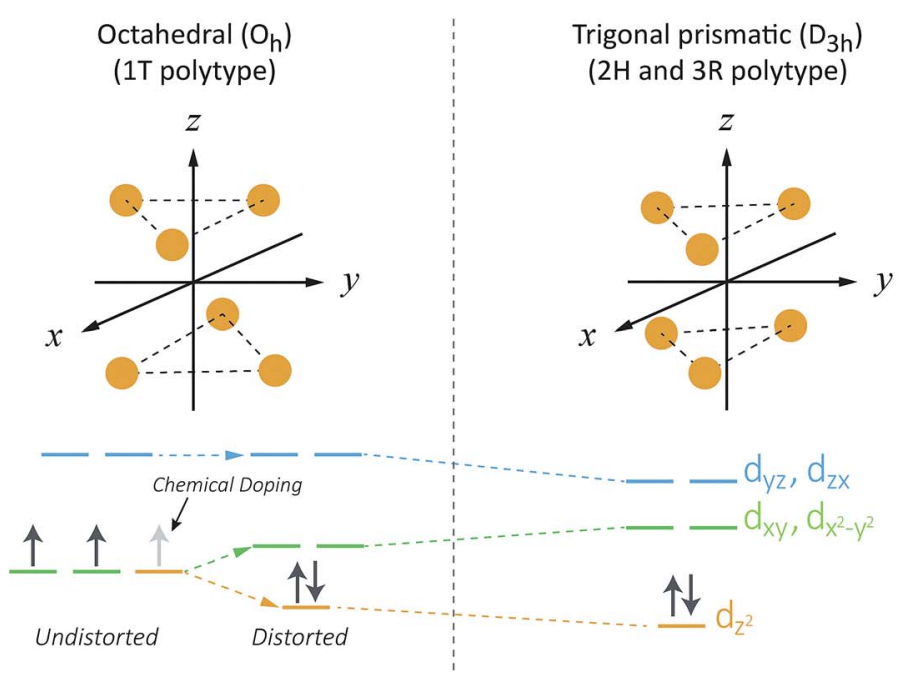

d

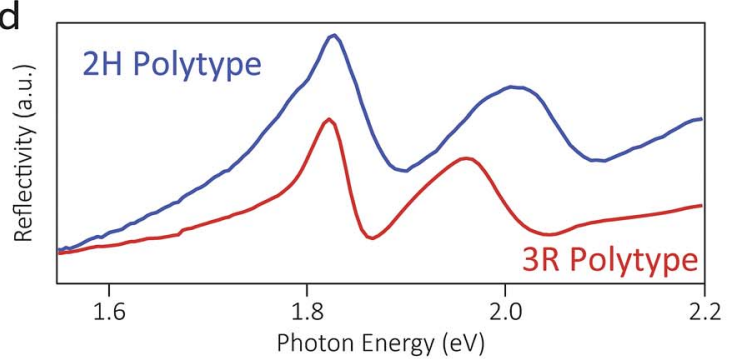

e

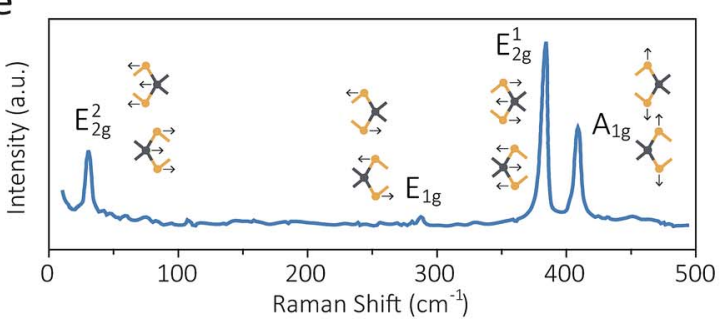

Fig. 2 (a) Schematic crystal structures of three polytypes - 1T, 2H, and 3R. The dashed lines show how the top views and the lateral views match with each other. In case of the $1 \mathrm{~T}$ and $2 \mathrm{H}$ polytypes, atoms of the underlying layers exactly overlap with the layers above. However, in case of the $3 \mathrm{R}$ polytype, some atoms of the underlying layers do not overlap with the above layers, and thus they are shown faint in the top view. (b) Schematic illustrations of the four possible structures of 1T polytype. ${ }^{85}$ The undistorted structure is identical to the top view of the 1T polytype in (a). Other three structures show the superstructures of distorted $1 \mathrm{~T}-\mathrm{MoS}_{2}$. Chemical bondings are omitted for clarity. Thick lines illustrate the boundary of a unit cell, and thin lines show the clustering of molybdenum atoms upon distortion. Note that the $2 a \times a$ superstructure is equivalent to the $\sqrt{3} a \times a$ superstructure. (c) Splitting Mo 4d orbitals under octahedral (1T polytype) and trigonal prismatic ligand field (2H and $3 \mathrm{R}$ polytype). ${ }^{85} \mathrm{Orange}$ coloured circles at the upper coordinates are sulphur atoms surrounding the omitted molybdenum atom at the origin of the coordinates. The colours of the orbital diagrams are unique to the orientation of the orbitals. The pale grey arrow at the orbital diagram of the undistorted octahedral ligand field shows the excess electron from doped alkali metals, which stabilises the undistorted octahedral ligand field. (d) Optical reflectivity of $2 \mathrm{H}$ - (blue) and 3R- $\mathrm{MoS}_{2}$ bulk (red). ${ }^{48}$ Two peaks near $1.8 \mathrm{eV}$ to $2.0 \mathrm{eV}$ correspond to A1 (lower energy) and B1 (higher energy) excitonic transitions. (e) Raman spectrum of bulk $2 \mathrm{H}-\mathrm{MoS}_{2}$. The corresponding normal vibration mode of each peak is shown. ${ }^{51}$ Reproduced with permission from: (b) and (c) ref. 85 (c) 2002 Elsevier; (d) ref. 48 @ 2014 Nature Publishing Group; (e) ref. 51 @ 1986 Springer.

preparation of $2 \mathrm{D} \mathrm{MoS}$ layers by means of mechanical exfoliation, chemical exfoliation and vapour-phase growth. The third part (Section 4 and 5) describes the physical properties of $2 \mathrm{D}$ atomic layers, focusing on their electronic (Section 4) and optoelectronic properties (Section 5). The final part (Section 6) describes the chemical reactivity of atomic layers of $\mathrm{MoS}_{2}$, specifically in terms of their reactivity with various chemical and biological species, as well as related applications. For convenience, the term "2D $\mathrm{MoS}_{2}$ " in this review stands for single- to few-layer forms of $\mathrm{MoS}_{2}$, where they have significantly different properties than the bulk form.

\section{Overview on bulk $\mathrm{MoS}_{2}$}

\subsection{Polytypes and crystallography}

As we have briefly discussed in Section 1, each layer of $\mathrm{MoS}_{2}$ is composed of S-Mo-S stacks, where a single molybdenum atom is surrounded by six sulphur atoms, as shown in Fig. 2a., ${ }^{2,15-17,34}$ $\mathrm{MoS}_{2}$ has three natural or synthetic polytypes of $\mathrm{MoS}_{2}$, which 
Table 1 Summary to properties of three polytypes of $\mathrm{MoS}_{2}$

\begin{tabular}{|c|c|c|c|c|}
\hline Polytype & Space group & Stacking order & Lattice parameters & Properties and remarks \\
\hline $1 \mathrm{~T}$ & $P 3 m 1$ & $\mathrm{AbC}$ & $a=5.60 \AA$ and $c=5.99 \AA$ (ref. 37) & $\begin{array}{l}\text { Paramagnetic. Metallic. Metastable structure } \\
\text { (spontaneous distortion forms superstructures) }\end{array}$ \\
\hline $3 R$ & $R 3 m$ & $\mathrm{AbA} \mathrm{BcB} \mathrm{CaC}$ & $a=3.17 \AA$ and $c=18.38 \AA$ (ref. 36) & $\begin{array}{l}\text { Diamagnetic. Indirect bandgap semiconductor } \\
\text { (ca. } 1.29 \mathrm{eV}) . \text { Its properties are similar to those of } \\
\text { the } 2 \mathrm{H} \text { polytype (ref. } 57 \text { ) }\end{array}$ \\
\hline
\end{tabular}

are $1 \mathrm{~T}, 2 \mathrm{H}$, and $3 \mathrm{R}$, depending on (1) the coordination of sulphur atoms with respect to the central molybdenum atom and (2) the stacking order of each layer (Fig. 2a and Table 1). ${ }^{34}$ Here the former number denotes the number of layers within a single crystallographic unit cell, and the latter letter describes the crystallographic structure, where $\mathrm{T}$ stands for trigonal, $\mathrm{H}$ for hexagonal, and $\mathrm{R}$ for rhombohedral structure. For example, a unit cell of the $2 \mathrm{H}$ polytype contains two layers that are centrosymmetric to each other (2), creating hexagonal structure $(\mathrm{H})$. Note that it is a convention to denote the polytype as the prefix to $\mathrm{MoS}_{2}$, if specified, as following: $2 \mathrm{H}-\mathrm{MoS}_{2}$ stands for the 2H polytype of $\mathrm{MoS}_{2}$.

Of these three polytypes, only $2 \mathrm{H}-\mathrm{MoS}_{2}$ and $3 \mathrm{R}-\mathrm{MoS}_{2}$ are stable and found in nature (see Table 1 for a list of their properties). ${ }^{34-36}$ Both the $2 \mathrm{H}$ and $3 \mathrm{R}$ polytypes commonly have trigonal prismatic coordination $\left(D_{3 h}\right)$ of molybdenum atoms, but they have different stacking order; $2 \mathrm{H}$ has stacking order of $\mathrm{AbA} \mathrm{BaB} \mathrm{AbA} . .$. , while that of $3 \mathrm{R}$ is $\mathrm{AbA} \mathrm{BcB}$ CaC AbA... Note that the upper and lower cases represent relative position of sulphur and molybdenum atoms, respectively. ${ }^{34}$ Nonetheless, both polytypes share crystallographic parameters. ${ }^{15,16,35,36}$ The distance between a Mo atom and its nearest $\mathrm{S}$ atom is $2.41 \AA^{15,35}$ The thickness of each layer is $3.15 \AA$, while the distance between adjacent layers is $3.49 \AA$, suggesting that the interlayer vdW interaction is weaker than intralayer covalent bonding.

In contrast, $1 \mathrm{~T}-\mathrm{MoS}_{2}$ is metastable, which is distinct from the aforementioned polytypes. ${ }^{37,38}$ It has octahedral coordination $\left(\mathrm{O}_{\mathrm{h}}\right)$ of molybdenum atoms, and its stacking order is AbC AbC... There are three reported types of distortions in $1 \mathrm{~T}-\mathrm{MoS}_{2}$, namely $2 a \times 2 a, \sqrt{3} a \times a$ and $\sqrt{3} a \times \sqrt{3} a$ superstructures. ${ }^{39}$ The phase transformation between these superstructures is related to the intercalation and oxidation level of alkali metal during synthesis, as we described later on Section 2.3 (Fig. 2b).

\subsection{The $2 \mathrm{H}$ and $3 \mathrm{R}$ polytypes with trigonal prismatic coordination}

Pristine $2 \mathrm{H}-\mathrm{MoS}_{2}$ is a diamagnetic semiconductor with indirect bandgap of $1.29 \mathrm{eV} .^{25,40,41}$ Mo $4 \mathrm{~d}$ orbitals are split by trigonal prismatic ligand field, and two d electrons of $\mathrm{Mo}^{4+}$ species occupy the lowest $4 \mathrm{~d}_{z^{2}}$ orbital, leading to diamagnetism and semiconductivity (Fig. 2c). ${ }^{42-45}$ Since the gap between the $d_{z^{2}}$ orbital (which mainly contributes to the valence band in the lattice) and $\mathrm{d}_{x y} / \mathrm{d}_{x^{2}-y^{2}}$ orbitals (which contributes to the conduction band in the lattice) is wide, a bandgap is formed even after the mixing with sulphur atoms occurs. ${ }^{\mathbf{4 0 , 4 2 - 4 6}}$ Detailed band structure studies reveals that the momentum of the valence band maximum (VBM) is different from that of the conduction band minimum (CBM), and thus it has an indirect bandgap (the calculated band diagram is shown in Fig. 5a, and detailed discussion is given in Section 5). Therefore, the indirect bandgap leads to a negligible quantum yield for the photoluminescence (PL). ${ }^{25}$ UV-Vis absorption spectrum has two prominent peaks at $1.9 \mathrm{eV}(\lambda \sim 640 \mathrm{~nm})$ and $2.1 \mathrm{eV}(\lambda \sim 580 \mathrm{~nm})$, which are often referred to as the $\mathrm{A} 1$ and $\mathrm{B} 1$ exciton absorption, respectively (Fig. 2d). ${ }^{47,48}$ A detailed explanation on the origin and characteristics of these peaks are given in Section 5.1. Raman scattering has four characteristic vibration modes: the $\mathrm{E}_{2 \mathrm{~g}}^{2}$ mode $\left(33 \mathrm{~cm}^{-1}\right.$, weak), $\mathrm{E}_{1 \mathrm{~g}}$ mode $\left(287 \mathrm{~cm}^{-1}\right.$, weak), $\mathrm{E}_{2 \mathrm{~g}}^{1}$ mode (383 $\mathrm{cm}^{-1}$, strong) and the $\mathrm{A}_{1 \mathrm{~g}}$ mode $\left(409 \mathrm{~cm}^{-1}\right.$, strong), which is shown in Fig. 2e. ${ }^{49-51}$

In terms of charge carrier transport, Hall measurements show that $2 \mathrm{H}-\mathrm{MoS}_{2}$ is an n-type semiconductor, with charge carrier mobility of $100 \mathrm{~cm}^{2} \mathrm{~V}^{-1} \mathrm{~s}^{-1}$ at room temperature. ${ }^{41}$ However, application of high pressure (ca. $60 \mathrm{GPa}$ ) on $2 \mathrm{H}-\mathrm{MoS}_{2}$ can induce a metallic state, along with change in Raman shifts due to the decrease in the interlayer distance and the change in the stacking order. ${ }^{52}$ Another viable way for semiconductor-tometal transition is alkali metal or alkali earth metal doping, in which case metal-to-superconductor phase transition further occurs at low temperature (refer to Section 2.3). ${ }^{53,54}$ For instance, Rb-doped $\mathrm{MoS}_{2}\left(\mathrm{Rb}_{0.3} \mathrm{MoS}_{2}\right)$ has a superconducting critical temperature of $6.9 \mathrm{~K}$.

$2 \mathrm{H}-\mathrm{MoS}_{2}$ is quite chemically inert. In vacuum, it is stable up to $1203{ }^{\circ} \mathrm{C}$, at which point it turns into $\mathrm{Mo}_{2} \mathrm{~S}_{3}{ }^{55}$ It does not dissolve in most solvents, but strong oxidizing reagents such as aqua regia may oxidize and dissolve it. ${ }^{15,56}$ It also reacts with oxygen to yield $\mathrm{MoO}_{3}$ around 400 to $600{ }^{\circ} \mathrm{C}$.

The physical and chemical properties of the $3 \mathrm{R}$ polytype is almost identical to those of the polytype, such as the Raman spectrum or the electronic band structure, except for minute differences in its band structure and UV-Vis absorption spectrum. ${ }^{16,47,48,57,58}$ For example, the A1 and B1 exciton absorption peaks are red-shifted by $10 \mathrm{meV}$ and $62 \mathrm{meV}$ respectively for 3R$\mathrm{MoS}_{2}$, since the different stacking order alters the ionic contribution within interlayer interaction (Fig. 2d). ${ }^{47,57,58}$

These two stable polytypes of $\mathrm{MoS}_{2}$ are found in nature, and they are mostly obtained through mining. The majority (ca. 
$80 \%$ ) of natural molybdenite has $2 \mathrm{H}$ stacking, and only $3 \%$ of the ore is purely $3 \mathrm{R}$ polytype, with the remnants exist as a mixture. ${ }^{59}$ Otherwise, they are artificially prepared through chemical synthesis, most importantly the chemical vapour transport (CVT) method, where a mixture of Mo, S, and halogen (iodine for $2 \mathrm{H}-\mathrm{MoS}_{2}$, and chlorine for $3 \mathrm{R}-\mathrm{MoS}_{2}$ ) is annealed at high temperature for several days to grow large single crystals. ${ }^{1,5,18,48}$ Selective chemical synthesis of $2 \mathrm{H}-\mathrm{MoS}_{2}$ is achieved by (1) the direct sulfidation of elemental Mo or its compounds at elevated temperature, ${ }^{15,60}$ or (2) the pyrolysis of $\left(\mathrm{NH}_{4}\right)_{2} \mathrm{MoS}_{4}$ and $\left(\mathrm{NH}_{4}\right)_{2} \mathrm{MoO}_{2} \mathrm{~S}_{2}$, which forms metastable intermediate $\mathrm{MoS}_{3}$ and $\mathrm{MoOS}_{2}$ respectively, all of which are reduced and crystallised to $2 \mathrm{H}-\mathrm{MoS}_{2}$ upon annealing at $350{ }^{\circ} \mathrm{C}$ or higher. ${ }^{61}$ Selective synthesis of $3 \mathrm{R}-\mathrm{MoS}_{2}$ is achieved by (1) the reaction of $\mathrm{MoO}_{3}$ and $\mathrm{S}$ in liquid $\mathrm{K}_{2} \mathrm{CO}_{3}$ at $900{ }^{\circ} \mathrm{C},{ }^{62}$ (2) the direct sulfidation of $\mathrm{Mo}$ under $4.7 \mathrm{GPa}$ at $1050{ }^{\circ} \mathrm{C},{ }^{63}$ or (2) the transformation of $2 \mathrm{H}-\mathrm{MoS}_{2}$ under 4 to $7.5 \mathrm{GPa}$ at $1900-2000{ }^{\circ} \mathrm{C} .{ }^{63}$ Note that reverse transformation takes place under vacuum at $1000{ }^{\circ} \mathrm{C} .{ }^{64}$

Due to its high stability and unique layered structures, $\mathrm{MoS}_{2}$, including both the $2 \mathrm{H}$ and $3 \mathrm{R}$ polytypes, has been widely used as a dry lubricant for several decades. ${ }^{\mathbf{1 8 6 5 - 6 9}}$ Similar to other layered materials, the in-plane friction coefficient for bulk $\mathrm{MoS}_{2}$ is much smaller (0.1) than that along $c$-axis $(0.26) .^{70}$ However, recent studies have discovered that the friction mechanism of $\mathrm{MoS}_{2}$ is distinct from other layered materials, where the lubrication of $\mathrm{MoS}_{2}$ is an intrinsic result of its inordinately weak vdW interlayer interaction. ${ }^{35,65,66,71,74}$ That is, lubrication of most layered materials requires the adsorption of vapour molecules, which can weaken the interlayer interaction. ${ }^{72,73}$ In case of some layered materials such as talc or muscovite mica, the interlayer interaction is so strong that they cannot even perform as lubricant. ${ }^{66,73}$ Therefore, most layered materials may not effectively lubricate in vacuum, where vapour molecules do not adsorb well. In contrast, friction in $\mathrm{MoS}_{2}$ decreases in vacuum, since adsorbents, especially oxidizing water, deter easy friction of weakly bound $\mathrm{MoS}_{2}$ layers. ${ }^{7,75}$ Therefore, $\mathrm{MoS}_{2}$ has been favoured as a lubricant under extreme conditions like vacuum systems or in spacecraft machinery. ${ }^{65,71}$

Another important field of industrial applications of $\mathrm{MoS}_{2}$ is the catalysis of sulphur reactions for the purpose of hydrodesulphurisation (HDS), i.e. substitution of the sulphur atom in organic molecules with a hydrogen atom. ${ }^{15,16,19,76,77}$ Usually supported on silica or alumina, $\mathrm{MoS}_{2}$ removes sulphur from various organic molecules containing sulphur such as thiophenes. Such activity specifically occurs at the rim edge of the $\mathrm{MoS}_{2}$ crystal, where sulphur vacancies easily adsorbs sulphur from the target molecule. ${ }^{76,78}$ A detailed mechanism study is given in Section 6.2.

\subsection{The 1T polytype with octahedral coordination}

$1 \mathrm{~T}-\mathrm{MoS}_{2}$, in contrast to the $2 \mathrm{H}$ and $3 \mathrm{R}$ polytypes, is metastable, metallic and paramagnetic because of the Mo atom's octahedral coordination. ${ }^{37,39,79}$ In order to synthesise $1 \mathrm{~T}-\mathrm{MoS}_{2}$, alkaliintercalated $\mathrm{MoS}_{2}$ is hydrated and then oxidized. ${ }^{37,79}$ This reaction exploits electron donation from alkali metal to the $\mathrm{MoS}_{2}$. As shown in Fig. 2c, an excess electrons from doped alkali metal stabilises octahedral coordination as in $\operatorname{ReS}_{2}$. The subsequent oxidation process removes the excess electron, but the coordination still retains as octahedral form with slight level of distortion. ${ }^{79}$

Notable consequences of the octahedral ligand field are the metallic conductivity and the metastable structure. As shown in Fig. 2c, two d electrons occupy triply degenerate d orbitals of the octahedral ligand field. ${ }^{38,46}$ Therefore, crystal distortion, similar to Jahn-Teller distortion or Fermi-surface nesting, takes place to break degeneracy and stabilise this metallic structure to some degree Equivalently, presence of excess electrons may stabilise the $1 \mathrm{~T}$ phase, as witnessed in Re-doped nanotubes and 2D atomic layers as well. ${ }^{38,39,80}$

Since the crystal distortion takes periodically, superstructures are formed. Diffraction and STM studies have found three types of superstructures at the intermediate and the final steps of the preparation process (Fig. 2b)..$^{39,81-84}$ At higher level of intercalation (i.e. lower level of oxidation; $\mathrm{K}_{x} \mathrm{MoS}_{2}, x \sim 0.7$ in case of $\mathrm{KMoS}_{2}$ ), triclinic or monoclinic structures of the $2 a \times 2 a$ superstructure are found. This $2 a \times 2 a$ superstructure are often found in other $1 \mathrm{~T}$ transition metal dichalcogenides with the $\mathrm{d}^{3}$ electronic configuration, like $\operatorname{ReS}_{2}{ }^{81,82}$ Further oxidation to $\mathrm{K}_{x}\left(\mathrm{H}_{2} \mathrm{O}\right)_{y} \mathrm{MoS}_{2}(x \leq 0.3)$ results in the metallic $\sqrt{3} a \times a$ (equivalently) superstructure, which is formed by zigzag chain-like clustering of Mo atoms. ${ }^{39,46}$ Note that this superstructure is also found in "restacked $\mathrm{MoS}_{2}$ ", which is the precipitation from the aqueous dispersion of lithiated $\mathrm{MoS}_{2}$ (we described the details of the preparation method of "restacked $\mathrm{MoS}_{2}$ " in Section 3.2). Further treatment of this superstructure with strong oxidising reagents such as $\mathrm{Br}_{2}$ forms the $\sqrt{3} a \times \sqrt{3} a$ superstructure by trimerisation of the Mo atoms. ${ }^{37,39,84}$ However, according to theoretical calculations, this superstructure has a small bandgap, and it is even more unstable than the $\sqrt{3} a \times a$ superstructure (refer to Section 4.1).

Therefore, metallic 1T- $\mathrm{MoS}_{2}$ does not have a precisely $\mathrm{d}^{2}$ electronic configuration. The $1 \mathrm{~T}-\mathrm{MoS}_{2}$ with the exactly $\mathrm{d}^{2}$ electronic configuration would instead favour the $\sqrt{3} a \times \sqrt{3} a$ superstructure and thus lose its metallic properties. Rather, this metallic $1 \mathrm{~T}-\mathrm{MoS}_{2}$ is equivalent to "restacked $\mathrm{MoS}_{2}$ ", and it has been insufficiently oxidised to have the $\sqrt{3} a \times a$ superstructure. ${ }^{84}$

Despite these distortions, $1 \mathrm{~T}-\mathrm{MoS}_{2}$ is still metastable and susceptible to the transformation to the $2 \mathrm{H}$ polytype. Differential scanning calorimetry (DSC) studies found that annealing up to $95{ }^{\circ} \mathrm{C}$ transforms the $1 \mathrm{~T}$ polytype into $2 \mathrm{H}$ polytype. Even simple aging is enough to induce the phase transformation to $2 \mathrm{H}$ polytype as well..$^{37,46,84}$

A well-known application of the metastable $1 \mathrm{~T}$ polytype is as an intermediate in intercalation chemistry. $\mathrm{A} \mathrm{MoS}_{2}$ lattice is an excellent intercalation host, because it accepts intercalates of different sizes, from small lithium ions up to macromolecules. ${ }^{\mathbf{8 1 , 8 5}}$ Stable $2 \mathrm{H}-\mathrm{MoS}_{2}$, however, does not accept most guest species directly, while $1 \mathrm{~T}-\mathrm{MoS}_{2}$ is active for the intercalation chemistry. Since lithium ion can directly intercalate into $2 \mathrm{H}$ $\mathrm{MoS}_{2}$ without pre-treatment as well as convert the inert $2 \mathrm{H}$ polytype into the active $1 \mathrm{~T}$ polytype, the intercalation of lithium ions, or lithiation, is done as matrix activation prior to 
intercalation of various guests species into inert $2 \mathrm{H}-\mathrm{MoS}_{2}$. A notable way of lithiation is refluxing a $2 \mathrm{H}-\mathrm{MoS}_{2}$ suspension with $n$-butyllithium (details are given in Section 3.2). ${ }^{85}$ An alternative way is creating an electrochemical cell comprised of $\mathrm{Li}$ and $2 \mathrm{H}-$ $\mathrm{MoS}_{2}$ as respective electrodes. ${ }^{86}$

Intercalation of other species then takes advantage of lithiation process by (1) exfoliation, where the lithiated $\mathrm{MoS}_{2}$, $\mathrm{LiMoS}_{2}$, colloid is hydrolysed to produce $\mathrm{Li}_{x}\left(\mathrm{H}_{2} \mathrm{O}\right)_{y} \mathrm{MoS}_{2}$ having the interlayer distance is $11.5 \AA$, or (2) restacking, which is treatment of $\operatorname{LiMoS}_{2}$ with an oxidising reagent or acid to obtain metallic $\sqrt{3} a \times a$ superstructures. ${ }^{39,84,85}$ These "activated" $\mathrm{MoS}_{2}$ can host various intercalates such as metallocene, ${ }^{87}$ metal clusters ${ }^{88}$ or polymers. ${ }^{89}$ Intercalation of these guests often changes the properties of $\mathrm{MoS}_{2}$; for instance, intercalation of conducting polymers such as polypyrrole (PPy) ${ }^{89}$ changes n-type $\mathrm{MoS}_{2}$ into a p-type semiconductor. Intercalation of an alkali metal makes $\mathrm{MoS}_{2}$ superconducting as well (refer to Section 2.2).

One of the major applications of $\mathrm{MoS}_{2}$ intercalation chemistry is as an electrode in a lithium ion battery (LIB). ${ }^{20,90} \mathrm{~A} \mathrm{MoS}_{2^{-}}$ based anode has higher specific capacity (907 $\mathrm{mA} \mathrm{h} \mathrm{g}^{-1}$ of reversible capacity after 50 cycles) than graphite (theoretical limit up to $372 \mathrm{~mA} \mathrm{~h} \mathrm{~g}^{-1}$ ), better rate capability $\left(554 \mathrm{~mA} \mathrm{~h} \mathrm{~g}^{-1}\right.$ for 20 cycles at $50 \mathrm{C}$ rate) than $\mathrm{Si}$ or $\mathrm{Ge}$ (in case of $\mathrm{Si}$, the theoretical specific capacity is $4212 \mathrm{~mA} \mathrm{~h} \mathrm{~g} \mathrm{~g}^{-1}$ at the initial lithiation, but it degrades rapidly upon repetitive charge and discharge ${ }^{91}$ ), and significantly less volumetric expansion during lithiation (3\% expansion upon full lithiation). ${ }^{\mathbf{9 0 , 9 2}}$ Its energy density is comparable or higher than conventional $\mathrm{LiCoO}_{2} \cdot{ }^{\mathbf{8 6}, 90} \mathrm{~A}$ typical problem of both $\mathrm{MoS}_{2}$ cathode and anode is their voltage, which is $2.0 \mathrm{~V}$ versus $\mathrm{Li} / \mathrm{Li}^{+}$. Since this voltage value is neither high (a high value is suitable for the cathode) nor low (a low value is suitable for the anode), such intermediate value reduces the net energy density. To this end, recent efforts have focused on overcoming this obstacle by forming $\mathrm{MoS}_{2}$ nanostructures or nanocomposites, which are well reviewed in the literatures. ${ }^{90}$

\section{Preparation of $2 \mathrm{D} \mathrm{MoS}_{2}$}

\subsection{Mechanical exfoliation and direct visualisation}

Conventional applications of $\mathrm{MoS}_{2}$, such as tribology or intercalation chemistry, are not much different from those of other layered materials. However, the isolation of single-layer $\mathrm{MoS}_{2}$ and the study on the nature of this atomically thin film have clearly distinguished this mineral from other layered materials because of its unique properties, most notably semiconductivity and valley polarisation. Preparation of $2 \mathrm{D} \mathrm{MoS}$ is therefore the first and the most crucial step to reveal and explore its nature.

The first report on single-layer $\mathrm{MoS}_{2}$ used mechanical exfoliation, i.e. repetitive cleavage of bulk crystals using adhesive tapes. ${ }^{10}$ Since molybdenite, especially $2 \mathrm{H}-\mathrm{MoS}_{2}$, is naturally occurring mineral (refer to Section 2.2), the preparation of single crystals does not require chemical vapour transport technique as required for other TMDs. ${ }^{\mathbf{1 0 , 1 5 , 2 5}}$ Thin film interference enables 2D MoS 2 visualisation on thin silicon oxide layers, with the best contrast achieved on either $90 \mathrm{~nm}$ or $270 \mathrm{~nm} \mathrm{SiO}_{2} /$
Si substrate, ${ }^{93,94}$ which is comparable to that of graphene (90 nm and $280 \mathrm{~nm}) .{ }^{95}$ In addition, the monochromatic red channel images enhances the contrast among different numbers of layers for a $300 \mathrm{~nm}$ oxide layer (Fig. $3 \mathrm{a}-\mathrm{c}$ ). ${ }^{\mathbf{9 6}}$

Despite the excellent quality of the exfoliated samples, their limited size of up to tens of micrometres and their poor thickness-control renders them apt only for fundamental research and for prototype device fabrication. While laser thinning technique can overcome the thickness variation locally, ${ }^{97}$ the development of chemical exfoliation and vapourphase growths have been significant steps in the ultimate goal of uniform, large-scale production.

\subsection{Chemical exfoliation for mass production of $2 \mathrm{D} \mathrm{MoS}_{2}$}

Chemical exfoliation, similar to the aforementioned mechanical exfoliation, peels off the layers of $2 \mathrm{H}-\mathrm{MoS}_{2}$ into 2D layers dispersed in a solvent. This approach is useful for the purpose of massive production and preparation of nanocomposites. ${ }^{\mathbf{9 8}}$

Early studies used the restacking process to chemically exfoliate $\mathrm{MoS}_{2}$, where lithium intercalation separates layers away from each other. ${ }^{99-101}$ When $\mathrm{MoS}_{2}$ is treated with strong reducing reagents containing lithium such as $n$-butyllithium or $\mathrm{LiBH}_{4}$, lithium ions intercalate into $\mathrm{MoS}_{2}$ and forms LiMoS 2 . While pristine $\mathrm{MoS}_{2}$ shows negligible dispersibility in water, $\mathrm{LiMoS}_{2}$ disperses quite well in water, forming dispersion of 2D $\mathrm{MoS}_{2}$ flakes. The dispersed flakes can be recovered by filtration or precipitation, and the resulting product is often called "restacked $\mathrm{MoS}_{2}$ ", because the layers are first exfoliated in dispersion and later "restacked" by filtration of the dispersion. A notable consequence of restacking process is the transformation of the $2 \mathrm{H}$ phase into the $1 \mathrm{~T}$ phase, as mentioned in Section 2.3. A scanning transmission electron microscopy (STEM) study has revealed that resulting sheets contain both the $1 \mathrm{~T}$ and $2 \mathrm{H}$ phases, forming coherent $2 \mathrm{H} / 1 \mathrm{~T}$ interface (Fig. 3d). ${ }^{102}$ In other words, the restacking process offers a facile way to synthesise two-dimensional $1 \mathrm{~T}-\mathrm{MoS}_{2}$ in large quantity, although the complete formation of the $1 \mathrm{~T}$ polytype is not achieved. ${ }^{102-104}$ Note that this restacked $\mathrm{MoS}_{2}$ is metallic due to the formation of the $\sqrt{3} a \times a$ superstructure. Additional annealing process is therefore required to convert all $1 \mathrm{~T}$ phase into the $2 \mathrm{H}$ phase.

In order to prepare $2 \mathrm{D} 2 \mathrm{H}-\mathrm{MoS}_{2}$ without any $1 \mathrm{~T}$ phase, the use of lithium should be avoided, and a number of reports used surfactants like sodium cholate instead. ${ }^{105,106}$ An aqueous dispersion of $\mathrm{MoS}_{2}$, with the proper surfactant, can be used for bioapplications such as described in Section 6.5, but in other applications remaining surfactants are often regarded as impurities.

Surfactant-free sonication of bulk crystal had problems with the stability of the exfoliated flakes, but it was recently discovered that using either certain good solvents or mixtures of poor solvents can resolve the problem; $N$-methyl-pyrrolidinone (NMP) and $N$-vinyl-pyrrolidinone (NVP) are typical examples of good solvents (Fig. 3e), while a mixture of ethanol and water is an example of the latter. ${ }^{\mathbf{1 1 , 1 0 7}}$ The fundamental role of these solvents is to stabilise the layers of $2 \mathrm{D} \mathrm{MoS}_{2}$ by 

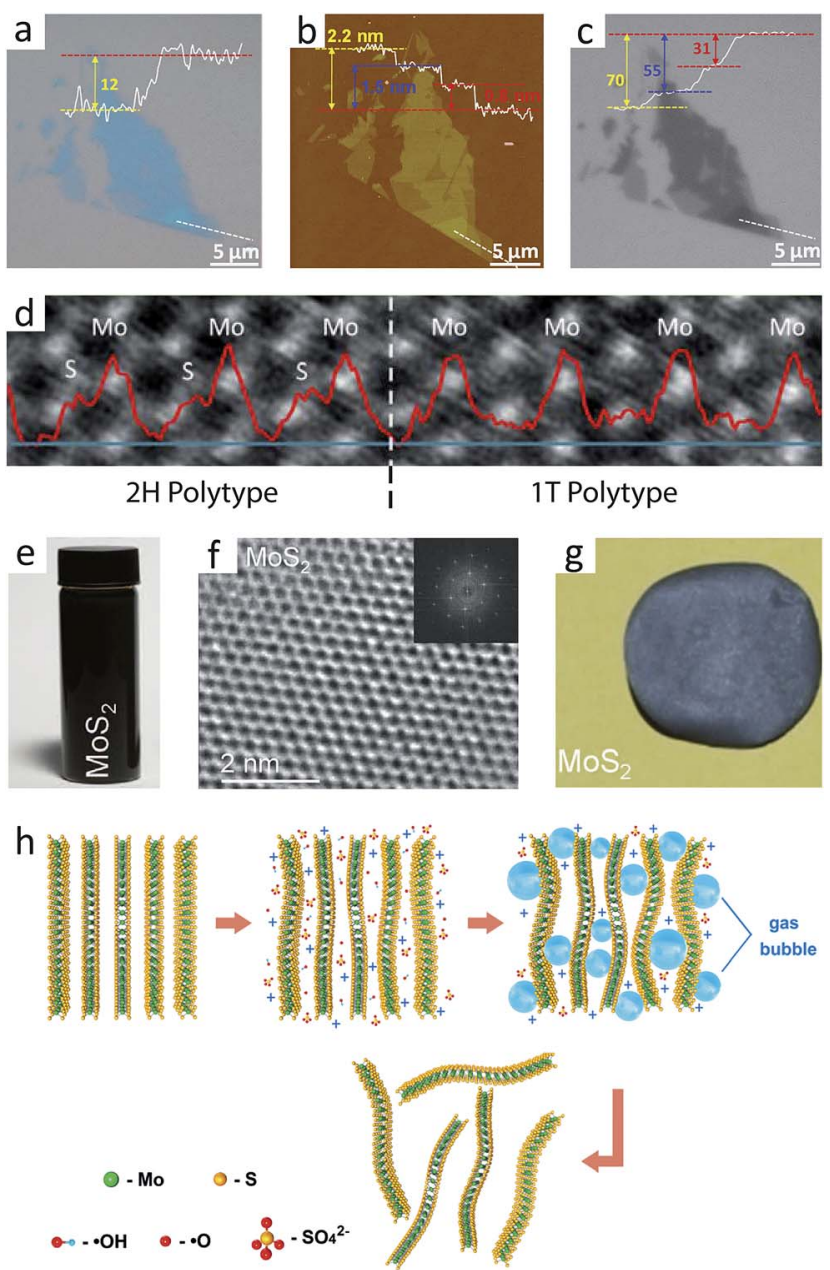

Fig. 3 (a)-(c): A mechanically exfoliated 2D $\mathrm{MoS}_{2}$ (1 to 3 layers) flake deposited on a $\mathrm{SiO}_{2}(300 \mathrm{~nm}) / \mathrm{Si}$ substrate, respectively observed by optical microscope (a), atomic force microscope (b), and optical microscope with red channels only (c).$^{96}$ Inset line graphs show the contrast ((a) and (c)) and thickness profiles (b) along the white dashed line. (d) A STEM image of chemically exfoliated 2D MoS 2 , showing the presence of both $2 \mathrm{H}$ and $1 T$ polytypes. ${ }^{102}$ The red graph shows the contrast of image along the blue line at the bottom. $2 \mathrm{H}$ and $1 \mathrm{~T}$ polytypes show the different pattern of contrast, in accordance with the crystallographic structures in Fig. 2a. (e)-(g): Dispersion of 2D 2H$\mathrm{MoS}_{2}$ atomic layers using a single solvent (e), its atomic structure measured by HRTEM (the inset shows its Fourier-transformed image) (f), and its free-standing film with thickness of ca. $50 \mu \mathrm{m}(\mathrm{g}){ }^{11}$ (h) A schematic illustration of electrochemical exfoliation. ${ }^{109} \mathrm{MoS}_{2}$ bulk is exfoliated by the bubbles from the electrolysis of water, and the exfoliated flakes are filtered. Reproduced with permission from: (a)-(c) ref. 96 (c) 2012 John Wiley and Sons; (d) ref. 102 ( 2012 American Chemical Society; (e)-(g) ref. 11 @ 2011 American Association for the Advancement of Science; (h) ref. 109 (c) 2014 American Chemical Society.

maximizing interaction between the solvent molecules and the layers of $2 \mathrm{D} \mathrm{MoS}$, such as London dispersion force, polar interaction or hydrogen bonding, because both types of solvents have high surface energy comparable to that of $2 \mathrm{D}$ $\mathrm{MoS}_{2}\left(c a .75 \mathrm{~mJ} \mathrm{~m}^{-2}\right){ }^{\mathbf{1 1 , 1 0 7 , 1 0 8}}$ High surface energy also prevents the exfoliated flakes from agglomeration. Absent of additional surfactants, the products of these methods provide high quality
2D flakes (Fig. 3f) and can be isolated as free-standing films (Fig. 3g).

Another viable approach for larger flakes is the adaptation of interlayer bubbling by electrochemical reaction (Fig. 3h). ${ }^{109}$ The principle guiding this process is interlamellar gas bubbling, which is also used in the restacking process. Electrochemical intercalation features high selectivity toward single layers with high quality and large size. However, this method results in a slight level of oxidation damage during the exfoliation process, though it can be cured by post-exfoliation annealing.

Chemical exfoliation methods produce $2 \mathrm{D} \mathrm{MoS}_{2}$ flakes in large quantity with the quality comparable to mechanically exfoliated ones. Demonstrably, chemically exfoliated samples show decent PL and comparable charge carrier mobility to mechanically exfoliated samples as well. ${ }^{110,111}$

This versatile approach, however, shares the same intrinsic limitations as most top-down methods; that is, it cannot produce a uniform, large-scale product on a solid substrate, since the lateral dimensions of each $2 \mathrm{D} \mathrm{MoS}$ flake are no larger than a few tens of micrometres, even under optimised condition. ${ }^{108,109,112}$ Additionally, some of solvents may contaminate the final products, and the film obtained by evaporating solvent is essentially a collection of discrete flakes rather than continuously connected film. In order to overcome these issues, a different way of preparation, i.e. the bottom-up approach, is required.

\subsection{Vapour-phase growth of large-scale 2D $\mathrm{MoS}_{2}$}

Vapour phase growth of $2 \mathrm{D} \mathrm{MoS}_{2}$ is one of the most well-known examples of a bottom-up approach. This method enables the growth of 2D $\mathrm{MoS}_{2}$ in large scale directly on top of solid substrates by means of chemical synthesis or physical assembly from gaseous precursors. This approach ultimately aims at scalability, uniformity, and the patternability of these continuous film product. Unlike mechanically exfoliated flakes, the films grown by vapour-phase growth contain ripples and grain boundaries, thus providing an interesting environment to study their properties. ${ }^{113}$ Note that all of the processes mentioned below produce the $2 \mathrm{H}$ (or trigonal prismatic configuration) polytypes.

Direct evaporation of $\mathrm{MoS}_{2}$ powder, i.e. vapour-solid growth, yields small flakes of high-quality single-layer $\mathrm{MoS}_{2}$ on solid substrates. ${ }^{30}$ This type of physical vapour deposition, however, cannot be used to grow large sheets of $2 \mathrm{D} \mathrm{MoS}_{2}$, leading researchers to pursue the most promising chemical synthesis methods using molybdenum and sulphur precursors.

Early studies used evaporation of molybdenum and sulphur precursors for the preparation of 2D $\mathrm{MoS}_{2}$ on metallic substrates in a scanning tunnelling microscope (STM) chamber. ${ }^{114,115}$ These products, however, are still discontinuous nanoflakes rather than continuous films. The first genuine example of a large-scale, continuous $2 \mathrm{D} \mathrm{MoS}$ film produced by vapour phase growth is the direct sulfidation of a pre-deposited thin molybdenum film (Fig. 4a and d). ${ }^{116}$ Although delicate control of reaction conditions yields uniform double-layer $\mathrm{MoS}_{2}$ in wafer scale $^{117}$ or even vertically aligned $\mathrm{MoS}_{2}$ films, ${ }^{118}$ 


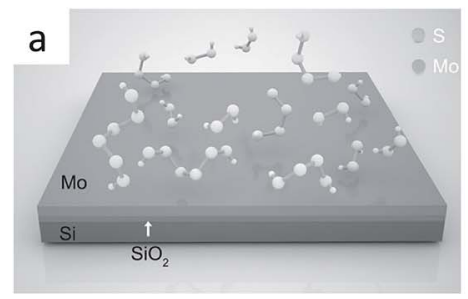

b
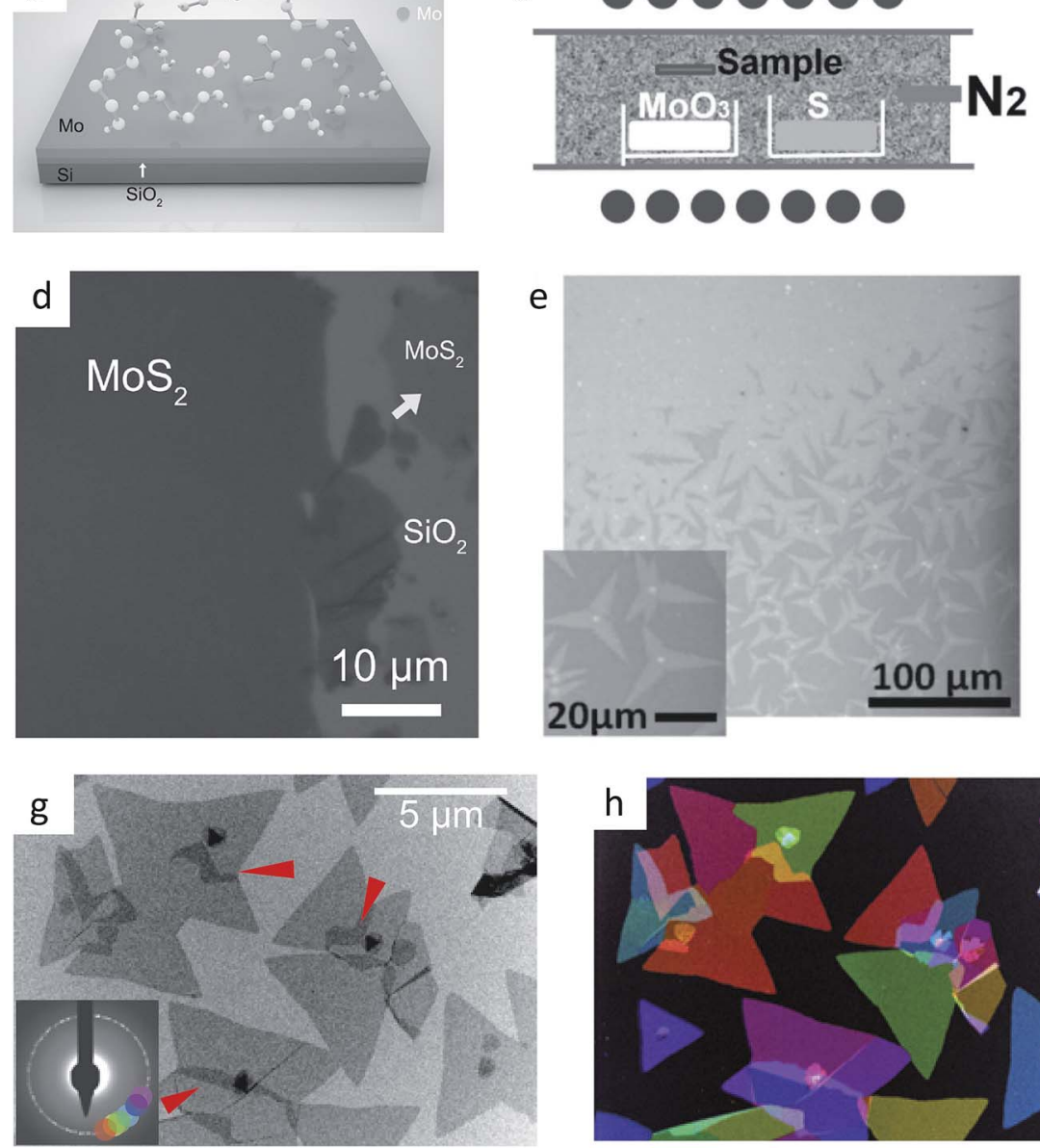
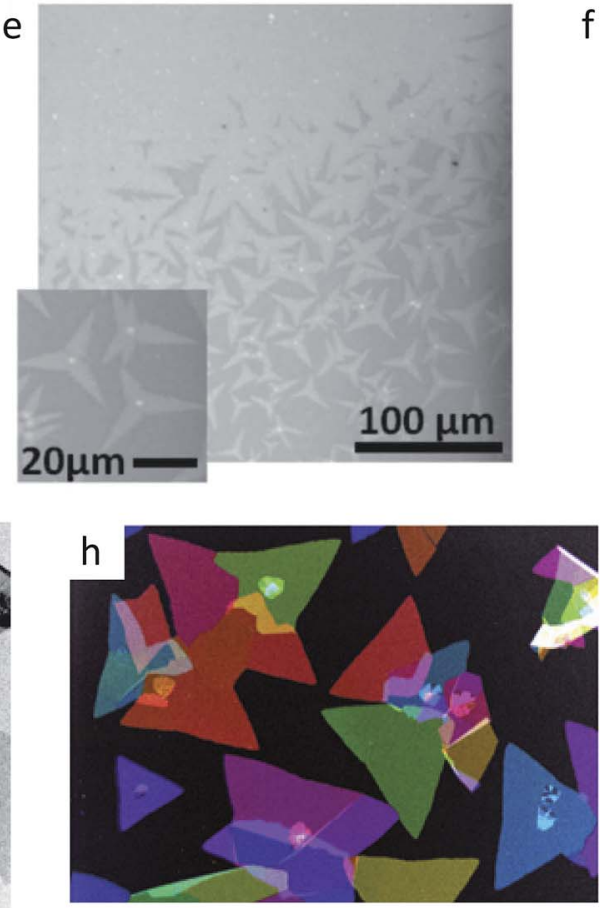

f
C
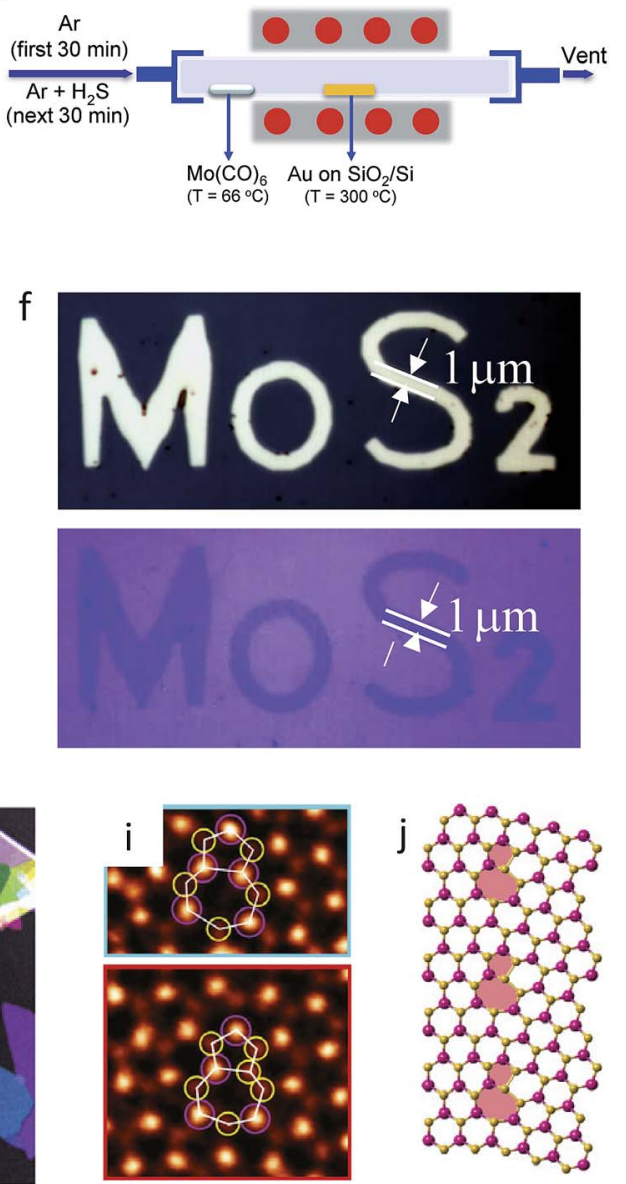

Fig. 4 (a) and (d): A schematic illustration of direct sulfidation of molybdenum film (a) and an optical image of its product (d).116 The white arrow indicates the region corresponding to single to double layers. (b) and (e): A schematic illustration of sulfidation of evaporated MoO 3 (b) and an optical image of its product, triangular crystal and film (e). ${ }^{122}$ (c) and (f): A schematic illustration of surface-alloy-assisted growth (c) and optical images of patterned Au film before reaction (upper) and the corresponding patterned 2D $\mathrm{MoS}_{2}$ atomic layers transferred to a $\mathrm{SiO} \mathrm{O}_{2}(285 \mathrm{~nm}) / \mathrm{Si}$ substrate (lower) (f). ${ }^{128}(\mathrm{~g})$ and (h): Typical $\mathrm{MoS}_{2}$ atomic layers grown by evaporation of $\mathrm{MoO}_{3}$ and subsequent sulfidation, observed by bright-field TEM (g) and the corresponding false-colour dark-field TEM image. In the dark-field TEM image, each colour shows a single grain having the diffraction pattern with the identical colour in the inset in (g). Note that red arrows indicate the overlaps of nearby crystals. ${ }^{129}$ (i) and (j): ADF-STEM images of the grain boundaries of CVD-grown polycrystalline single-layer $\mathrm{MoS}_{2}$ (i) and the schematic structure of a grain boundary of polycrystalline single-layer $\mathrm{MoS}_{2}$ (j). ${ }^{132}$ Reproduced with permission from: (a) and (d) ref. 116 @ 2012 John Wiley and Sons; (b) and (e) ref. 122 ○ 2012 John Wiley and Sons; (c) and (f) ref. 128 @ 2014 John Wiley and Sons; (g) and (h) ref. 129 @ 2013 Nature Publishing Group; (i) and (j) ref. 132 @ 2013 Nature Publishing Group.

Stranski-Krastanov mechanism, which governs the physical deposition of the molybdenum film, hinders uniform growth over a large area. ${ }^{119}$ The preparation of molybdenum precursor film with uniformity is therefore the key issue in the synthesis of 2D $\mathrm{MoS}_{2}$ over large scale.

To this end, chemical reactions that have been used to synthesise bulk $2 \mathrm{H}-\mathrm{MoS}_{2}$ (refer to Section 2.2) have been adapted to prepare 2D films. One example is the pyrolysis of $\left(\mathrm{NH}_{4}\right)_{2} \mathrm{MoS}_{4}$. Vapour-phase injection of $\left(\mathrm{NH}_{4}\right)_{2} \mathrm{MoS}_{4}$ dissolved in $N, N$-dimethylformamide (DMF) results in the van der Waals epitaxy of $2 \mathrm{D} \mathrm{MoS}$ flakes on graphene. ${ }^{120}$ Upon prolonged reaction, these 2D $\mathrm{MoS}_{2}$ flakes grow and merge together to produce a rugged film. This graphene/ $\mathrm{MoS}_{2}$ heterostructure, however, has limited applicability, since such semiconducting layers that are inseparable from its metallic substrate cannot be utilised for device fabrication. In contrast, dip-coating $\left(\mathrm{NH}_{4}\right)_{2} \mathrm{MoS}_{4}$ on an insulating substrate resolves this problem to grow transferrable, high-crystalline three-layer $\operatorname{MoS}_{2}{ }^{121}$ Although the quality is comparable to mechanically exfoliated samples, the necessity of high temperature annealing $\left(1000{ }^{\circ} \mathrm{C}\right)$ and the poor controllability over the number of layers (mainly three layers, with partial two layers) are the main limitations of this two-step reaction.

Evaporation of volatile $\mathrm{MoO}_{3}$ on an arbitrary substrate followed by sulfidation process is another example that utilises bulk synthesis reaction (Fig. $4 \mathrm{~b}$ and e). ${ }^{122,123}$ This process effectively grows $\mathrm{MoS}_{2}$ atomic layers by coalescence of triangularly shaped single crystals, which altogether results in largescale, few-layer $\mathrm{MoS}_{2}$ displaying a high level of crystallinity (Fig. 4e). Note that a more precise control of the number of layers, including single layers, can be achieved by use of 
hygroscopic $\mathrm{MoCl}_{5}$ instead of $\mathrm{MoO}_{3},{ }^{124}$ or sulfidation of predeposited inert $\mathrm{MoO}_{2}$ followed by exfoliation. ${ }^{125}$ This catalystfree growth can be applied on arbitrary substrates, but provision of nucleation seeds by surface treatment with perylene3,4,9,10-tetracarboxylic acid tetrapotassium salt (PTAS) enhances its versatility. ${ }^{\mathbf{1 2 6}}$ In addition, certain substrates are known to show unique properties due to the interaction between $2 \mathrm{D} \mathrm{MoS}_{2}$ and the substrate. Mica is such an example; since $\mathrm{MoS}_{2}$ and mica has a lattice mismatch, the single-layer $\mathrm{MoS}_{2}$ directly grown on mica has an intrinsic compressive strain. ${ }^{127}$ Therefore, the PL from this single-layer $\mathrm{MoS}_{2}$ on mica has enhanced PL intensity and suppressed PL helicity (refer to Section 5.1 and 5.2). Note that the original PL properties are recovered when the $2 \mathrm{D} \mathrm{MoS}$ grown on mica is transferred onto a $\mathrm{SiO}_{2}$ substrate. Despite these attractive properties of the product, the process of evaporation of volatile molybdenum compound followed by sulfidation, however, lacks patternability due to random nucleation of precursor. Scalability is another limitation, since the lateral growth, which increases the size of the product, often competes with the vertical growth, which increases the number of layers, especially under prolonged reaction.

A recent paper has reported a novel route that uses surface alloy of Mo and Au as the molybdenum precursor (Fig. 4c), where the surface alloy provides uniform, atomically thin Mo layer that is confined to the surface of immiscible Au film. ${ }^{128}$ This Mo layer selectively reacts with $\mathrm{H}_{2} \mathrm{~S}$ to form $\mathrm{MoS}_{2}$. This method features specific growth of atomic layers on top of Au film only, which can be easily patterned prior to reaction (Fig. 4f). However, the resulting 2D $\mathrm{MoS}_{2}$ film exhibits low carrier mobility and small grain sizes, which should be overcome.

Vapour-phase-grown 2D $\mathrm{MoS}_{2}$ exhibits various kinds of defects such as adlayers or grain boundaries (GB) (Fig. $4 \mathrm{~g}$ and h). Adlayers on single-layer $\mathrm{MoS}_{2}$ are quite visible with optical or electron microscopy. They are found in the middle of the $\mathrm{MoS}_{2}$ flake due to the excessive growth or near the crystal edge due to overlap of neighbouring layers. ${ }^{\mathbf{1 2 9 , 1 3 0}}$ These adlayers resemble double layers in terms of the structure, but they have higher conductivity than $\mathrm{MoS}_{2}$ itself owing to excess carrier density. ${ }^{\mathbf{1 3 0}}$ In contrast, GBs are not directly visualised by optical or electron microscopy; they can be visualised by dark-field transmission electron microscopy (DF-TEM) because of the different crystal orientation of each grain. ${ }^{\mathbf{1 3 1}}$ While certain grains may overlap with adjacent grains to form double layers, most grains coalescence and fuse with their neighbours to form an atomic boundary. Since pristine $2 \mathrm{H}-\mathrm{MoS}_{2}$ has hexagonal rings of Mo-SMo-S-Mo-S (Fig. 2a), GBs exhibit chains of defective rings, such as five- and seven-membered rings or even four- and eightmembered rings along their GB (Fig. $4 \mathrm{i}$ and j). ${ }^{\mathbf{1 2 9 , 1 3 2 - 1 3 4}}$ The type of GB is determined by the abundance of either sulphur or molybdenum during growth reaction as well as the angle between the grains. ${ }^{\mathbf{1 2 9 , 1 3 3}}$ These grain boundaries often show electrical band structures distinct from pristine $2 \mathrm{D} \mathrm{MoS}$, such as formation of mid-gap states, or n-doping in case of excess molybdenum atoms. ${ }^{133}$ Electrical transport across mirror grain boundaries is almost indistinguishable from that of pristine plane, but the transport across the tilted grain boundaries shows a drop in electrical conductivity. ${ }^{129,132,134}$ Theoretical calculations also predict that chains of four-membered rings from $60^{\circ}$ grain boundary would show metallic states..$^{133}$

Studying the synthesis of large-scale $2 \mathrm{D} \mathrm{MoS}_{2}$ is important not only for the realisation of the vast potentials of $2 \mathrm{D} \mathrm{MoS}$ but also for the related synthesis of other TMDs or heterostructures made of various $2 \mathrm{D}$ nanomaterials. Nonetheless, a way to synthesise single-crystalline single layers in wafer scale (i.e. larger than four inches) on arbitrary substrates is still yet to be found. In addition, the selective growth of 2D 1T-MoS ${ }_{2}$ film in large scale has still not been achieved yet; currently, lithium intercalation can only produce $1 \mathrm{~T}$ phase in small flakes, ${ }^{\mathbf{8 4 , 1 0 3 , 1 0 4}}$ and electron dosing by irradiation of electron beams can locally induce the phase transition from $2 \mathrm{H}$ to $1 \mathrm{~T}$ phases in Re-doped single-layer $\mathrm{MoS}_{2} \cdot{ }^{\mathbf{8 0}}$

\section{Charge carrier transport of 2D $\mathrm{MoS}_{2}$ and applications to transistor devices}

\subsection{Electronic band structure}

One of the first studies on $2 \mathrm{D} 2 \mathrm{H}-\mathrm{MoS}_{2}$ that have triggered such popularity, as shown in Fig. 1, is the discovery of semiconductivity with direct bandgap of $1.9 \mathrm{eV}$ (Fig. 5a) and characterisation of prototype transistor devices..$^{25,26,135}$ This was a significant discovery because such behaviour is impossible for graphene, the first 2D nanomaterial to be isolated; graphene is a native semi-metal that demands complex processes to open up a bandgap. ${ }^{136}$

Such direct bandgap transition, which is one of the key characteristics of $2 \mathrm{D} \mathrm{MoS}_{2}$, is attributed to the contributions of atomic orbitals to the electronic band states at different points in momentum space. ${ }^{25,135,137,138}$ As shown in Fig. 5a, the bulk form of $2 \mathrm{H}-\mathrm{MoS}_{2}$ exhibits the indirect-gap transition from the valence band maximum (VBM) at the $\Gamma$ point to the conduction band minimum (CBM) between the $K$ point and the $\Gamma$ point. The orbitals contributing to the conduction band states at the $K$ point state are localised within the S-Mo-S sandwich, but the states at the $\Gamma$ point and the CBM point have strong characters of the d orbitals of Mo and the antibonding $\mathrm{p}_{z}$ orbitals of $\mathrm{S}$, leading to strong dependence on vertical interlayer coupling. As the number of layers decreases, the $K$ point state remains nearly constant, but the energy of the other states is reduced. To this end, when the number of layers is minimised (i.e. a single layer), the $\Gamma$ point state in the valence band has less energy than that of the $K$ point, and thus the $K$ point state become the VBM. Also, the state between the $K$ point and the $\Gamma$ point, where the bulk CBM is located, has higher energy than that of the $K$ point state in the conduction band. Therefore, single-layer $2 \mathrm{H}-\mathrm{MoS}_{2}$ shows the direct gap transition at the $K$ point.

Nonetheless, all of these states are still nearly degenerate in single layers, and thus a small degree of changes in the orbital overlap will alter the transition. Indeed, upon application of tensile stress the fundamental transition in single layers becomes indirect bandgap transition between $\Gamma$ and $K$ points. ${ }^{139-141}$ 
a
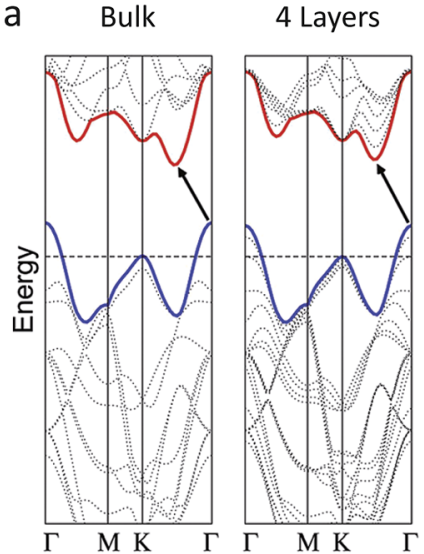

$\mathrm{b}_{\mathrm{y}}$

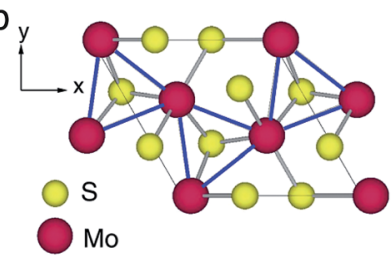

2 Layers

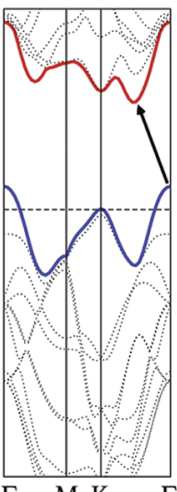

C

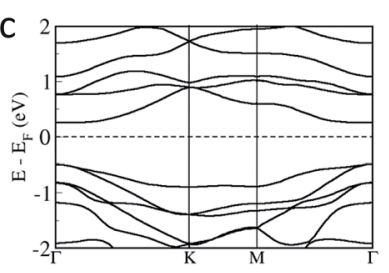

Fig. 5 (a) Calculated electronic band structures of bulk, four-layer, two-layer, and single-layer $\mathrm{MoS}_{2}$ (from left to right). ${ }^{135}$ Black arrows indicate the fundamental transitions. The horizontal dashed lines demonstrate the energy of the highest occupied states at the $K$ point, which becomes the VBM in single layers. As the number of layers decrease, the energy at the $I$ point reduces, and it becomes lower than $K$ point state in single layers, leading to direct gap transition. (b) and (c): The superstructure of the distorted $1 T$ polytype single layer, showing trimerisation of Mo atoms (blue lines) (b), and the calculated bands structure of single-layer $1 \mathrm{~T}-\mathrm{MOS}_{2}$ with superstructure, showing semiconducting bandgap of $0.7 \mathrm{eV}$ (c) ${ }^{142}$ Reprinted with permission from: (a) ref. 135 (c) 2010 American Chemical Society; (b) and (c) ref. 142 (c) 2014 American Physical Society.

Electronic properties of the $1 \mathrm{~T}$ single layer are unique in that they are predicted to display a bandgap opening of $0.7 \mathrm{eV}$ (Fig. 5c) due to distortion from centrosymmetric (ideal) structure to trimerisation of molybdenum atoms having the $\sqrt{3} a \times \sqrt{3} a$ superstructure (Fig. 5b). ${ }^{142}$ Such trimerisation has been predicted and observed in bulk layers as well (refer to Section 2.3). Berry phase calculation shows that the distorted structure results in spontaneous polarisation along the $z$ axis, while the in-plane polarisation vanishes. The coercive electric field required for switching the sign of polarisation domain is estimated to be $10^{7} \mathrm{~V} \mathrm{~cm}^{-1}$, which is easily achieved by a fieldeffect transistor (FET) device. That is, if this phase can be stabilised, single-layer $1 \mathrm{~T}-\mathrm{MoS}_{2}$ is an excellent candidate for the thinnest ferroelectric device ever.

\subsection{Charge carrier transport properties}

In order to take full advantage of semiconducting properties of 2D $2 \mathrm{H}-\mathrm{MoS}_{2}$, in-depth studies on its semiconducting nature and properties is the first step, and a number of charge transport studies have been carried out so far.

A typical back-gated FET with single-layers $\mathrm{MoS}_{2}$ as channel shows charge carrier mobility of 0.5 to $3 \mathrm{~cm}^{2} \mathrm{~V}^{-1} \mathrm{~s}^{-1}$ at room temperature with n-type semiconductivity. ${ }^{10}$ Introduction of an additional top gate with high- $\kappa$ dielectric material such as $\mathrm{HfO}_{2}$, as shown in Fig. 6a, may enhance the charge carrier mobility and transport characteristics (Fig. 6b). ${ }^{26,143-146}$ Such an effect is also valid for few-layer $\mathrm{MoS}_{2}$ FET, stating that in spite of its indirect bandgap, few-layer $\mathrm{MoS}_{2}$ is still an attractive semiconductor material. ${ }^{147}$

Chemical doping is often an effective way to modulate electric transport properties in semiconductors. In general, the charge carrier mobility in $2 \mathrm{D} \mathrm{MoS}_{2}$ is robust against ionic doping; to induce a significant effect, the required carrier density is at least $5 \times 10^{11} \mathrm{~cm}^{-2} .{ }^{146}$ Higher doping of $\mathrm{Nb}$, a wellknown p-type dopant for $\mathrm{MoS}_{2}$ bulk-, ${ }^{41}$ fullerene-, ${ }^{148}$ and nanotube ${ }^{149}$-type of $\mathrm{MoS}_{2}$, turns $2 \mathrm{D} \mathrm{MoS}$ into p-type semiconductor with hole mobility of $8.5 \mathrm{~cm}^{2} \mathrm{~V}^{-1} \mathrm{~s}^{-1} \cdot{ }^{150} \mathrm{Au}$ is another example of p-dopants, ${ }^{151}$ which results in p-type transport from surfacealloy assisted growth. ${ }^{128}$ Re is an n-type dopant for $\mathrm{MoS}_{2}$ fullerene and nanotubes, ${ }^{152}$ but theoretical study predicts that it may not be effective for $2 \mathrm{D}$ layers. ${ }^{153}$ Alkali metal is a better option; exposure of FET device into $\mathrm{K}$ vapour results in electron concentration up to $1 \times 10^{13} \mathrm{~cm}^{2}{ }^{153,154}$ It was also shown that the $\mathrm{Na}$ impurities or the $\mathrm{O}$ dangling bonds from a $\mathrm{SiO}_{2}$ substrate can become n-type and p-type dopants, respectively. ${ }^{155}$
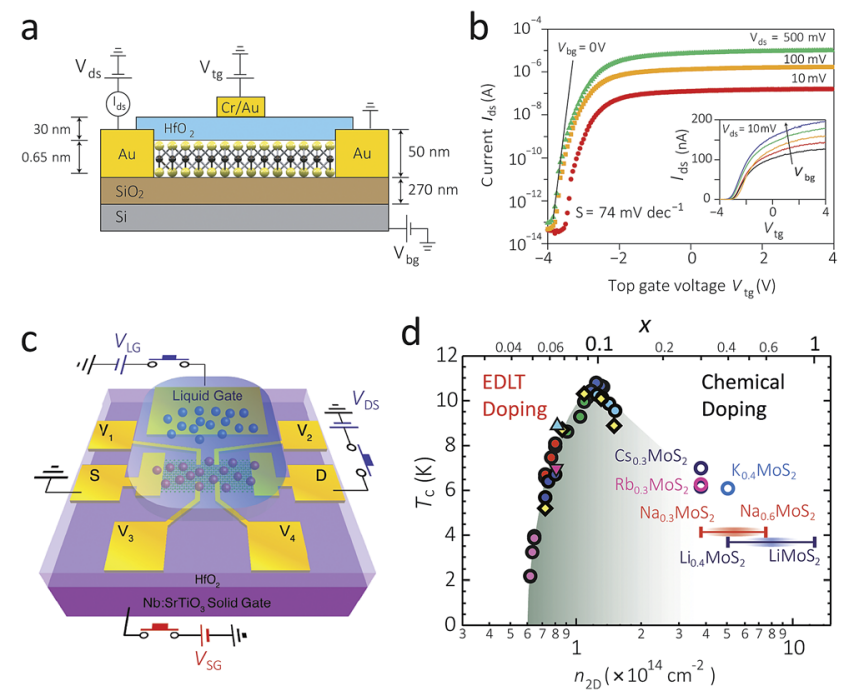

Fig. 6 (a) and (b): A schematic illustration of a 2D MoS 2 FET device with both the back gate $\left(\mathrm{SiO}_{2}\right)$ and the top gate $\left(\mathrm{HfO}_{2}\right)(\mathrm{a})$, and its transfer characteristics with the varying top-gate voltage (b). ${ }^{26}$ Since the increase in the top gate voltage makes more current flow, it is an ntype semiconductor. The inset in (b) shows that the increasing backgate voltage also increases the electric current. (c) A schematic illustration of an EDLT with an ionic liquid gate. Here the transparent blue droplet shows the ionic liquid, which is located between the channel at the centre and the gate electrode at the top. ${ }^{157}$ (d) The phase diagram of the superconductor critical temperature versus the charge carrier concentration. The green area, which looks like a dome, represents the superconducting state. The marks at the left half shows the data obtained by EDLT doping, and those at the right half shows that of chemical doping by alkali metal. ${ }^{157}$ Reproduced with permission from: (a) and (b) ref. 26 (c) 2011 Nature Publishing Group; (c) and (d) ref. 157 (c) 2012 American Association for the Advancement of Science. 
Electrical doping provides a reversible way to modulate the charge carrier transport properties of $2 \mathrm{D} \mathrm{MoS}_{2}$. While a mild level of electrical doping induces the gate effect just as in typical semiconductors, a higher level of doping, with charge carrier density more than $1 \times 10^{13} \mathrm{~cm}^{2}$, induces semiconductor-tometal transition due to electron-electron interactions. ${ }^{156}$

A more extreme case of electrical doping by the electric double-layer transistor (EDLT) device with an ionic liquid gate (Fig. 6c) induces switchable, native superconductivity, i.e. without chemical doping (refer to Section 2.2). ${ }^{157,158}$ Fabrication of prototype devices demonstrates that the critical temperature of superconductivity can reach up to $10.8 \mathrm{~K}$ upon optimal electric doping, which is the highest value among all TMD materials. A combined study of both chemical doping and electrical doping shows that the superconducting state appears within a specific "dome-like" range of the temperature and the charge carrier density, where the critical temperature is maximised at carrier density of $1.2 \times 10^{14} \mathrm{~cm}^{-2}$ (Fig. 6d). ${ }^{157}$

\subsection{Toward fabrication of high-performance atomically thin FET}

In order to fully exploit the aforementioned properties, it is essential to optimise the FET fabrication conditions are crucial as well. Typical electrode materials for $2 \mathrm{D} \mathrm{MoS}_{2}$ FET are Au (work function $=5.0 \mathrm{eV}$ ) and $\mathrm{Ti}$ (work function $=4.3 \mathrm{eV}$ ); $\mathrm{Ti}$ is a better option for Ohmic-like contact, since its work function is comparable to $\mathrm{MoS}_{2}$ (electron affinity $=4.0 \mathrm{eV}$ ), and Ti has a smaller lattice mismatch (by $1 \%$ ) with $\operatorname{MoS}_{2} \cdot{ }^{26,159}$ A more comprehensive study shows that an even better option is rare Sc (work function $=3.5 \mathrm{eV}$ ). ${ }^{\mathbf{1 6 0}} \mathrm{Mo}$ is another option, where the contact region becomes metallic, resulting in a small Schottky barrier of $0.1 \mathrm{eV} .^{161}$

In terms of gate dielectrics, the fabrication of an FET device with both the bottom and top gates can enhance the 2D $\mathrm{MoS}_{2}$ FET performance, leading to a high mobility of almost 15 to 55 $\mathrm{cm}^{2} \mathrm{~V}^{-1} \mathrm{~s}^{-1}$, an on-off ratio more than $10^{10}$, and a subthreshold swing reaching down to $60 \mathrm{mV}$ per decade at an extremely short channel (Fig. 6b)..$^{\mathbf{2 6 , 1 4 4 , 1 4 5 , 1 6 2}}$ Note that special cares should be taken for the calculation of the mobility value of such dual-gate devices, since the capacity of the gate dielectrics may be underestimated, which results in the overestimation of the charge carrier mobility value. ${ }^{\mathbf{4 1 , 1 4 4 , 1 4 5}}$ Such performance enhancement by dual gate fabrication is attributed to suppressed charged-impurity scattering, in accordance with theoretical calculations. ${ }^{\mathbf{1 4 3 , 1 4 5 , 1 5 6}}$ In addition, the use of $\mathrm{HfO}_{2}$ is also favoured over $\mathrm{Al}_{2} \mathrm{O}_{3}$ in flexible devices, because it features slow crack propagation and robust charge carrier mobility against bending. ${ }^{163}$

Special modification of device components further demonstrates novel properties of $2 \mathrm{D} \mathrm{MoS}_{2}$. For instance, the introduction of the previously mentioned EDLT device with an ionic liquid gate, which enables extremely high doping $\left(\sim 1 \times 10^{14}\right.$ $\mathrm{cm}^{2}$ ), can establish a p-type conducting state, leading to ambipolar behaviour of few-layer $\mathrm{MoS}_{2}{ }^{164}$ Using PMMA as gate dielectric and capping material can further enhance charge carrier mobility. ${ }^{165}$ Also, applying different bias between source- gate and drain-gate in the EDLT device with an ionic liquid gate allows for a stable $\mathrm{p}-\mathrm{n}$ junction within a single crystal of $\mathrm{MoS}_{2}$, when the temperature is changed from $220 \mathrm{~K}$ to $180 \mathrm{~K}$ while the gate voltage is applied. ${ }^{\mathbf{1 6}}$ This phenomenon is based on cooling across the glass transition temperature of the ionic liquid with simultaneous gating, where the motion of the ions of the ionic liquid couples with the accumulation of charges. Incorporation with light illumination or adsorption of small molecules can further tune transistor operation, suggesting utilisation as a phototransistor or a gas sensor, as described in Section 5.4 and 6.1 , respectively.

Although the relatively low charge carrier mobility of $2 \mathrm{D}$ $\mathrm{MoS}_{2}$ discourages direct competition with III-V semiconductors, 2D $\mathrm{MoS}_{2}$ transistor devices show high on-off ratios and low stand-by power, which suggests suitable application for low-power electronics. ${ }^{162}$ As a proof of concept, a group of single-layer $\mathrm{MoS}_{2}$ transistors have successfully demonstrated logic gate operations, which are the key functionality required for the fabrication of complex processing units. ${ }^{167}$

\section{Optical behaviours of 2D MoS 2 and applications to optoelectronic devices}

\subsection{Absorption and emission characteristics of 2D $\mathrm{MoS}_{2}$}

Direct bandgap makes the optoelectronics of single layer $2 \mathrm{H}$ $\mathrm{MoS}_{2}$ exceptional among all types of $\mathrm{MoS}_{2}$. The reflectance difference spectrum has two prominent peaks at $670 \mathrm{~nm}$ (A1) and $627 \mathrm{~nm}$ (B1), similar to the bulk form, but it shows strong emission corresponding to these absorption peaks (Fig. 7a), with quantum yield of $4 \times 10^{-3}$, which is four orders of magnitude higher than that of the bulk. ${ }^{25,135}$ These emissions are in fact PL generated from direct bandgap transition. Doublelayer $\mathrm{MoS}_{2}$ shows one PL peaks at $1.59 \mathrm{eV}(\mathrm{ca} .780 \mathrm{~nm})$ and two peaks near $1.88 \mathrm{eV}$ ( $c a .660 \mathrm{~nm}$ ), with quantum yield around $10^{-5}$ to $10^{-6} \cdot{ }^{25}$ These peaks shift with the increasing number of layers, where the first peak at $1.59 \mathrm{eV}$ continuously approaches to indirect bandgap of $1.2 \mathrm{eV}$ in the bulk. Band structure studies show that the first peak is indirect-gap luminescence, while the others are direct gap hot luminescence, i.e. the reverse process of the $\mathrm{A} 1$ and $\mathrm{B} 1$ excitonic absorption. ${ }^{25}$

The splitting of the A1 and B1 peaks is mainly attributed to the splitting of the VBM at the $K$ point, which has different origins in the single layer case and the multilayer case. ${ }^{168}$ On the one hand, splitting of the VBM in single layer is purely attributed to spin-orbit coupling because of the lack of inversion symmetry. On the other hand, splitting in multilayers, which do possess inversion symmetry, is due to a combination of both spin-orbit coupling and interlayer coupling.

Since absorption and emission profiles are closely related to the band structure, modification of the band structure may change the intensity and energy of the PL peaks. For instance, electrochemical intercalation of alkali metal ions can reversibly quench $\mathrm{PL}$ in 2D $2 \mathrm{H}-\mathrm{MoS}_{2}$, because it induces the phase transformation to the metallic $1 \mathrm{~T}$ phase. ${ }^{169}$ Also, the application of strain reduces the intensity and energy of A1 exciton peak, since the strain will reduce the direct gap at $K$ point, as 

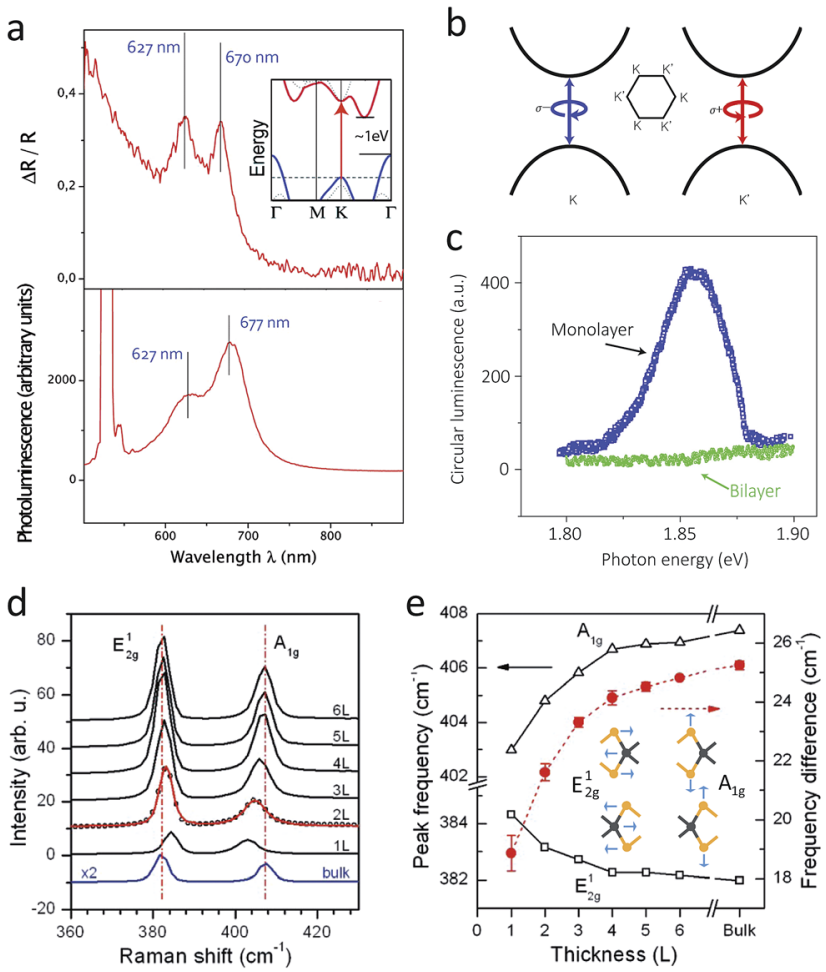

Fig. 7 (a) A reflectance difference (upper) and photoluminescence spectrum (lower) of single-layer $\mathrm{MoS}_{2}$. The inset in the upper graph describes the band structure of bulk MoS, having indirect bandgap ${ }_{2} \cdot{ }^{135}$ (b) and (c): A schematic band diagram showing the origin of valley polarisation (b) and unique circular dichroism of photoluminescence from single layer at $10 \mathrm{~K}$ under circularly polarised illumination (c). ${ }^{28}$ (d) and (e): Raman spectra of $\mathrm{MoS}_{2}$ with different numbers of layers (d) and shift of Raman vibration modes with respect to the different number of layers (e). The inset in (e) depicts corresponding normal vibration modes. ${ }^{173}$ Reproduced with permission from: (a) ref. 135 (c) 2010 American Chemical Society; (b) and (c) ref. 28 (c) 2012 Nature Publishing Group; (d) and (e) ref. 173 (c) 2010 American Chemical Society.

discussed in Section 4.1. ${ }^{139-141}$ Note that the strain required for direct-to-indirect gap transition in optical bandgap is much less than that of the electrical bandgap due to the large exciton binding energy.

\subsection{Unique valley polarisation and trion formation}

A notable feature of PL from single-layer $\mathrm{MoS}_{2}$ is valley polarisation, where the PL conserves the circular dichroism of the incident light. The unique phenomenon is the results of spinorbit coupling together with the absence of inversion symmetry. ${ }^{27-29}$ Single-layer $\mathrm{MoS}_{2}$ has six CBMs and VBMs, which are referred as "valleys", at the $K$ points (refer to Section 4.1). Spin-obit coupling further splits each valley into different spins (refer to Section 5.1), and circularly polarised light excites opposite spins at the $\mathrm{K}$ and $\mathrm{K}^{\prime}$ valleys located at the opposite positions in $k$-space (Fig. 7b). Here, because of time reversal symmetry, single layer $\mathrm{MoS}_{2}$, which lacks inversion symmetry, does not allow inter-valley transition. This spin-selective population at opposite valleys is preserved upon the polarised illumination, and thus PL maintains the same circular dichroism as the incident light (Fig. 7c). Since this phenomenon requires the broken inversion symmetry and the spinorbit coupling at the same time, even double-layer $2 \mathrm{H} \mathrm{MoS}_{2}$ does not show valley polarisation. However, the $3 \mathrm{R}$ polytype shows valley polarisation regardless of the number of layers, since the structure inherently lacks inversion centres. ${ }^{48}$ Note that illuminating a single-layer $\mathrm{MoS}_{2}$ transistor with circularly polarised light results in valley Hall effect, where Hall voltage occurs due to valley polarisation. ${ }^{\mathbf{1 7 0}}$

Another unique feature of the optoelectronics of single-layer $\mathrm{MoS}_{2}$ is the formation of a negative trion, a quasiparticle comprised of two electrons and one hole, upon illumination. ${ }^{171}$ Such phenomenon is absent in other conventional direct-gap semiconductors, where illumination only creates an exciton, a pair of an electron and a hole. Gate-voltage-dependent absorption and PL studies reveal the existence of two components in both PL and A1 exciton absorption in single-layer $\mathrm{MoS}_{2}$. The intensity of the higher energy component diminishes upon electron doping, but the other component largely retains its intensity regardless of the applied gate voltage. Here the former is attributed to the formation of neutral excitons, while the latter is related to trions, which are created by binding of electrons to pre-existing electron-hole pairs. Its binding energy is large enough (20 meV) to manifest at room temperature as well. One of the direct results of trions is the effect of chemical doping on the intensity of PL; for instance, p-type dopants like tetracyanoquinodimethane (TCNQ) enhance the PL intensity, because it promotes recombination of excitons rather than trions. ${ }^{172}$

\subsection{Raman spectroscopy and phonon-photon interaction}

Phonon-photon interaction of 2D $\mathrm{MoS}_{2}$ features the shift of vibration energy upon the decreasing number of layers. Of the four major Raman-active vibration modes, out-of-plane (or equally $\mathrm{A}_{1}^{\prime}$ mode in single layers, concerning their different symmetry from the bulk) and in-plane (or equally $\mathrm{E}^{\prime}$ mode in single layers) exhibit notable shifts. ${ }^{173}$ The $\mathrm{A}_{1 \mathrm{~g}}$ vibration mode experiences softening, since interlayer coupling is weakened upon the decreasing number of layers. In contrast, the $\mathrm{E}_{2 \mathrm{~g}}^{1}$ vibration mode shows anomalous stiffening, which is attributed to the stronger dielectric screening of the long-range Coulombic interaction with the decreasing number of layers. ${ }^{\mathbf{1 7 4}}$ To this end, in single-layer $\mathrm{MoS}_{2}$, the $\mathrm{A}_{1 \mathrm{~g}}$ vibration appears near $404 \mathrm{~cm}^{-1}$ (cf. $408 \mathrm{~cm}^{-1}$ for bulk), and the $\mathrm{E}_{2 \mathrm{~g}}^{1}$ mode is near $385 \mathrm{~cm}^{-1}$ ( $c f$. $383 \mathrm{~cm}^{-1}$ for bulk), regardless of the wavelength of the incident laser (Fig. 7d). ${ }^{\mathbf{1 7 3 , 1 7 5}}$ Therefore, the energy difference between them is often a facile indicator of the number of layers (Fig. 7e).

In addition, the position of each vibration mode with respect to the zero-strain reference reflects the amount of applied strain. ${ }^{139-141}$ While the out-of-plane $\mathrm{A}_{1 \mathrm{~g}}$ mode is robust against tensile strain, the in-plane $\mathrm{E}_{2 \mathrm{~g}}^{1}$ mode significantly changes. At low strain, the in-plane $\mathrm{E}_{2 \mathrm{~g}}^{1}$ vibration mode is softened, and thus the corresponding peak in Raman spectrum is red-shift and slightly broadened. However, at higher strain, the crystal symmetry is apparently changed, and thus the double 
degeneracy of the $\mathrm{E}_{2 \mathrm{~g}}^{1}$ vibration mode is lifted, leading the splitting into two peaks in the Raman spectrum.

The aforementioned peaks are first-order Raman peaks, which are commonly found regardless of the wavelength of incident laser. ${ }^{175}$ However, $325 \mathrm{~nm}$ laser reveals additional resonant Raman spectrum within the range of 750 to 840 $\mathrm{cm}^{-1} \cdot{ }^{176}$ These peaks are attributed to spin-orbit splitting at the valence band, specifically because of electron-two-photon triple resonance via Fröhlich-interaction and deformation potential. Note that the energies of these peaks do not depend on the number of layers. Also, $633 \mathrm{~nm}$ laser induces resonant Raman peaks such as $2 \mathrm{LA}(\mathrm{M})$ peaks near $440 \mathrm{~cm}^{-1}$ in thicker $\mathrm{MoS}_{2} \cdot{ }^{175}$

\subsection{Application to optoelectric device}

The direct bandgap of single-layer $\mathrm{MoS}_{2}$ results in unique photon-electron interaction. Therefore, characterisation of FET devices under external illumination offers a versatile way to assess the optoelectric nature of $2 \mathrm{D} \mathrm{MoS}_{2}$. When light with lower energy than the direct bandgap illuminates the interface between an electrode and 2D $\mathrm{MoS}_{2}$, it shows photothermoelectric effect. ${ }^{177}$ This is unique among semiconductors in that other materials do not flow photocurrent upon sub-bandgap illumination. This is attributed to the large mismatch of Seebeck coefficients between the electrodes and the single-layer $\mathrm{MoS}_{2}$, with values ranging from $-4 \times 10^{2}$ to $-1 \times 10^{5} \mu \mathrm{V} \mathrm{K}^{-1}$ depending on the gate voltage.

Upon super-bandgap illumination, single-layer $\mathrm{MoS}_{2}$ phototransistors show photoresponsivity of $7.5 \mathrm{~mA} \mathrm{~W}^{-1}$ up to $880 \mathrm{~A} \mathrm{~W}^{-1}$ for optimised device geometry, on-off ratio of $10^{3}$ and switching time of $50 \mathrm{~ms} .{ }^{\mathbf{1 7 8 , 1 7 9}}$ Ultrahigh photoresponsivity is six orders of magnitude higher than that of graphene, because the existence of a bandgap hinders the exciton recombination. Moreover, it is sensitive to most of the visible light because of its direct bandgap, and its noise-equivalent power is $1.8 \times 10^{-15} \mathrm{~W}$ $\mathrm{Hz}^{-1 / 2}$, which is an order less (better) than commercial silicon avalanche photodiodes. Such application is also demonstrated for vapour-phase-grown $\mathrm{MoS}_{2}$, in which case the photoresponsivity is up to $2200 \mathrm{~A} \mathrm{~W}^{-1}$ and the time constant is down to $55 \mathrm{~ms} .{ }^{180}$ Bandgap modulation by thickness control results in tuning of the photoresistivity wavelength; for example, singlelayer and double-layer $\mathrm{MoS}_{2}$ are sensitive to green light, and triple-layer $\mathrm{MoS}_{2}$ is sensitive to red light. ${ }^{181}$

Based on the photon-electron interaction discussed above, $2 \mathrm{D} \mathrm{MoS}_{2}$ can be also incorporated into photovoltaic cells as the active layer in a heterojunction solar cell ${ }^{182}$ or as a holeextraction layer. ${ }^{183}$ The former (ref. 182) has a structure of $\mathrm{Al} /$ 2D $\mathrm{MoS}_{2} /$ p-type Si/Ag, where n-type $\mathrm{MoS}_{2}$ and p-type Si form type-II heterojunction. Its photoconversion efficiency is $5.23 \%$, which is the highest among all single-layer TMD-based solar cells. In case of the latter (ref. 183), its band structure, chemical stability and lack of dangling bonds make $2 \mathrm{D} \mathrm{MoS}$ suitable for photovoltaic cell operation, and it shows power conversion efficiency of $8.11 \%$, which is superior to the previously reported $\mathrm{MoO}_{3}$ counterpart.

Electroluminescence is another fundamental optoelectric operation of direct bandgap semiconductors, which is required for light emitting devices. Electroluminescence of $2 \mathrm{D} \mathrm{MoS}_{2}$ is demonstrated using hot carrier process at a Schottky barrier. ${ }^{\mathbf{1 8 4}}$ This hot carrier process occurs when excited electrons are injected into the conduction band through the Schottky barrier, where significant band bending generates excitons. These excitons are recombined through a radiative process, which enables electroluminescence without the formation of a $\mathrm{p}-\mathrm{n}$ junction. This electroluminescence has prominent A1 excitonic characteristics (at $685 \mathrm{~nm}$ ) only, because B1 excitonic states are not populated upon electric bias. Pristine $\mathrm{MoS}_{2}$, however, still requires higher quantum yield for practical application, and this is expected to be achieved by formation of heterostructures.

Based on these novel electronic and optoelectronic properties, 2D $\mathrm{MoS}_{2}$ is now one of the most promising materials for flexible, transparent electronic device components. For instance, an AFM study has proved its substantially high mechanical stiffness (Young's modulus of $270 \pm 100 \mathrm{GPa}$, and breaking stress of $22 \pm 4 \mathrm{GPa}$ ). That is, it is flexible enough to be used with polyimide (PI) or polydimethylsiloxane (PDMS) substrate, which is one of the most frequently used substrates for flexible electronics. ${ }^{185,186}$

\section{Molecular and biomolecular interactions of 2D MoS 2}

\subsection{Application to molecular and biomolecular sensors}

The study of the interaction between 2D $\mathrm{MoS}_{2}$ and other molecules often utilise FET devices, especially in case of the semiconducting $2 \mathrm{H}$ polytype, because these devices offer an appropriate environment to control and assess electron transport upon chemical modification of $2 \mathrm{D} \mathrm{MoS}_{2}$. That is, reversible adsorption of various moieties on $2 \mathrm{D} \mathrm{MoS}_{2}$ often changes its properties as well as the properties of the target molecules. Detection of such changes open up the possibility of developing 2D $\mathrm{MoS}_{2}$ based sensors. In addition, flexibility, transparency, economic massive production, and high sensitivity are major advantages of $2 \mathrm{D} \mathrm{MoS}$ over other bulk or other nanomaterials, even including graphene and other 2D nanomaterials. ${ }^{\mathbf{1 1 , 3 3 , 9 8 , 1 8 7}}$

One such example is as an active sensing component for chemical and biomolecular sensors. Adsorption of target molecules changes the charge transport properties of the $2 \mathrm{D}$ $\mathrm{MoS}_{2}$ FET device, so the electric conductivity correspondingly changes upon exposure. One of the first demonstrations is a NO gas sensor. ${ }^{33}$ This device shows remarkable detection power, with a detection limit which reaches down to $0.8 \mathrm{ppm}$ and a high signal-to-noise ratio (Fig. 8a). A more comprehensive study shows that other molecules like $\mathrm{NO}_{2}, \mathrm{NH}_{3}$, and $\mathrm{H}_{2} \mathrm{O}$ are well detected, and its detection power can be further enhanced by the incorporation of gate effect or light illumination. ${ }^{\mathbf{1 8 8}}$ For example, a moderate level of illumination of green light significantly enhances the detection sensitivity of $\mathrm{NO}_{2}$. A larger number of layers is favoured for better gas detection, since thicker samples have higher charge carrier concentration and higher on/off ratio. A first-principle calculation study has shown that this change of current is caused by the charge transfer from the adsorbent gas to the $2 \mathrm{D} \mathrm{MoS}_{2}$ film. ${ }^{188,189}$ Upon adsorption of 

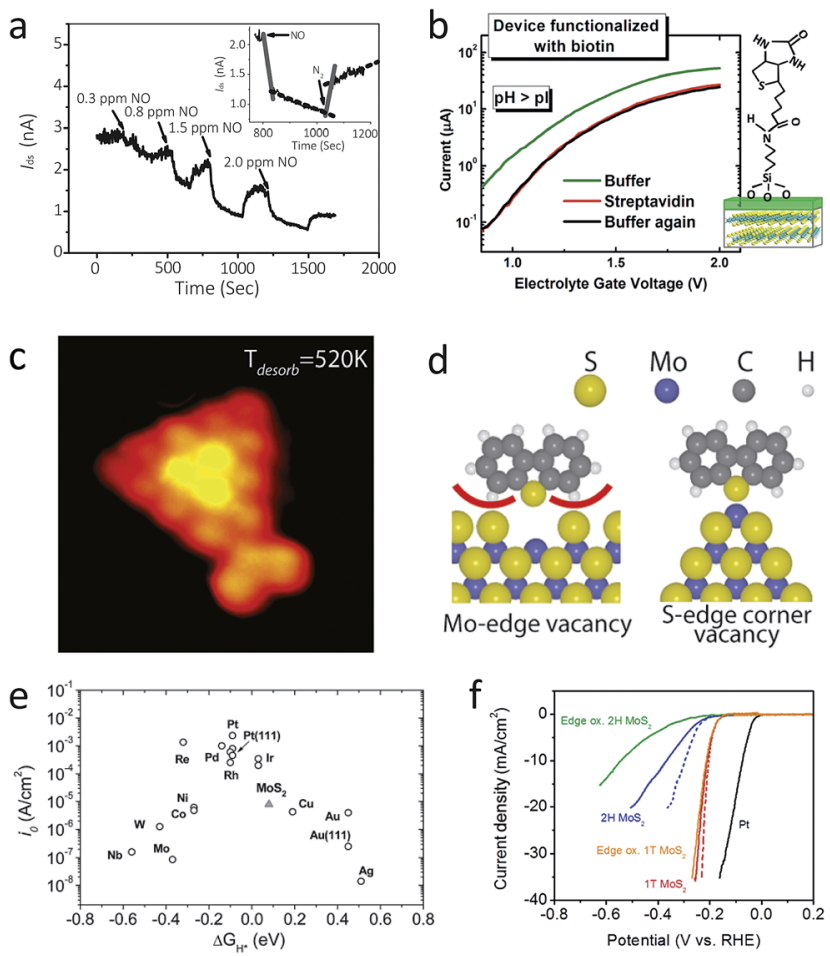

Fig. 8 (a) The current change of a double-layer $\mathrm{MoS}_{2}$ transistor upon exposure of $\mathrm{NO}$ gas. The inset shows that the current level recovers upon purging with $\mathrm{N}_{2}$ gas. ${ }^{33}$ (b) Electron transport characteristics of $\mathrm{MoS}_{2}$-based biosensor with biotin decoration (left) and the schematic illustration of the device (right). ${ }^{187}$ (c) and (d): A STM image of $\mathrm{MoS}_{2}$ with thiophene adsorbed at the corner (c) and its molecular scheme showing the preference of S-edge corner vacancy for thiophene adsorption (d). ${ }^{191}$ (e) A volcano plot of various metal electrodes for HER catalysis, including $\mathrm{MoS}_{2} \cdot{ }^{31}$ (f) Polarisation curve of $1 \mathrm{~T}-\mathrm{MoS}_{2}, 2 \mathrm{H}-\mathrm{MoS}_{2}$ (before and after edge oxidation), and Pt electrode. ${ }^{104}$ While edge oxidation significantly reduces the catalytic efficiency of $2 \mathrm{H}-\mathrm{MoS}_{2}$ (blue to green), it does not affect 1T-MoS 2 much (red to orange). Reproduced with permission from: (a) ref. 33 (c) 2012 John Wiley and Sons; (b) ref. 187 @ 2014 American Chemical Society; (c) and (d) ref. 191 (c) 2010 American Chemical Society; (e) ref. 31 (c) 2007 American Association for the Advancement of Science; (f) ref. 104 (c) 2013 American Chemical Society.

the gas molecules, the Fermi level of $2 \mathrm{D} \mathrm{MoS}$ changes without significant changes in the band structure due to charge transfer. In addition, most of the gas molecules, including $\mathrm{NO}^{33}$ are charge acceptors, which turns $2 \mathrm{D} \mathrm{MoS}_{2}$ into p-type semiconductor, and $\mathrm{NH}_{3}$ is shown to be a charge donor.

Another proposed application of $2 \mathrm{D} \mathrm{MoS} 2$ FET is a label-free sensor for $\mathrm{pH}$ and protein. ${ }^{187}$ When a top-gated $2 \mathrm{D} \mathrm{MoS} 2$ FET is immersed in an electrolyte, which can behave as a top gate electrode, the surface of the gate oxide is naturally decorated with $\mathrm{OH}$ moieties. Therefore protonation (low $\mathrm{pH}$ ) or deprotonation (high $\mathrm{pH}$ ) changes surface potential of the gate oxide, resembling gate effect. For example, a lower level of $\mathrm{pH}$ results in electron doping. Further decoration by biotin can detect streptavidin, a protein that can combine with biotin quite well, with a detection limit reaching down to $100 \mathrm{fM}$ (Fig. 8b).

Unlike the aforementioned applications, where $2 \mathrm{D} \mathrm{MoS}_{2}$ is an active channel of an FET device, $2 \mathrm{D} \mathrm{MoS}_{2}$ itself can be utilised as a sensor for single-stranded DNA (ssDNA). That is, the basal plane of single-layer $\mathrm{MoS}_{2}$ is a good adsorbent for ssDNA via van der Waals interactions..$^{190}$ When FAM(fluorescein amidite; a type of fluorescent dye)-labelled DNA was mixed with the single-layer $\mathrm{MoS}_{2}$ in dispersion, ssDNAs adsorb on the $\mathrm{MoS}_{2}$ flakes, leading to the quenching of fluorescence. However, upon injection of a complementary strand, the fluorescence was turned on again, since the ssDNA is detached from the surface of the $2 \mathrm{D} \mathrm{MoS}$ flakes. A similar experiment was successfully carried out for aptamers, where the detection limit was $5 \mu \mathrm{M}$.

\subsection{Surface chemistry: HDS catalysis and mechanism}

Direct utilisation of 2D $\mathrm{MoS}_{2}$ for chemical studies takes advantage of its similarity to bulk in terms of chemical reactivity. Therefore, it is now possible to directly observe and establish models for atomic changes during conventional surface chemistry reaction, by means of standard microscopic tools. One such study is HDS catalysis as described in Section 2.2. A recent STM-based study revealed that catalysis activity is maximised for S-terminated small clusters, where some of the corner sulphur atoms are easily removed to leave vacant sites. These vacant sites show least steric effect toward the chemisorption of thiophenes (Fig. 8c and d). ${ }^{191}$ In accordance with this observation, several attempts have been made to improve catalytic efficiency, i.e. (1) the substitution of edge sulphur with $\mathrm{Ni}$ or Co "promoters", where sulphur atoms will adsorb on the S-Mo-Ni or -Co terminal edges, ${ }^{78}$ and (2) the formation of nanoparticles or nanoclusters, where the high exposure of rim sites improves catalytic activity. ${ }^{192}$

\subsection{Electrochemistry: catalyst for hydrogen evolution reaction}

While most chemical properties of $2 \mathrm{D} 2 \mathrm{H}-\mathrm{MoS}_{2}$ are quite similar to bulk, still it has certain distinct and unique properties. Hydrogen evolution reaction (HER), or production of hydrogen by electrolysis of water, is one of such examples, which holds promising applicability, ${ }^{31,32}$ where the pristine bulk $\mathrm{MoS}_{2}$ behaves as a less efficient HER catalyst. ${ }^{14,45,193}$ The role of $2 \mathrm{D} 2 \mathrm{H}-\mathrm{MoS}_{2}$ here is as an electrode; protons $\left(\mathrm{H}^{+}\right)$are adsorbed on the metallic edges of $2 \mathrm{D} 2 \mathrm{H}-\mathrm{MoS}_{2}$, where electrons transferred to reduce the protons into hydrogen gas. A STM study has shown that S-bonded Mo edges are active for HER, and the total length of edge has linear correlation with the HER catalytic efficiency. To this end, 2D 2H-MoS 2 is located at the middle of the volcano plot, which states high exchange current density and reversible hydrogen adsorption $(\Delta G \sim 0)$ (Fig. 8e). Nonetheless, pristine $\mathrm{MoS}_{2}$ has low adsorption ratio (ca. 25\%) on the edge sites and poor electric conductivity that hinders kinetics, resulting in, for instance, a Tafel slope of 55 to $60 \mathrm{mV}$ per decade. In order to improve this, vertically aligned $\mathrm{MoS}_{2}$ layers are used to create intentionally large exposure of edge sites. ${ }^{118}$ Incorporation with conductor provides another viable strategy to improve electron transfer kinetics, and examples include reduced graphene oxide $(\mathrm{rGO})^{194}$ and epitaxially grown metal nanostructures. ${ }^{195}$ For instance, $\mathrm{Pt}-\mathrm{MoS}_{2}$ hybrid has Tafel slope 
of $40 \mathrm{mV}$ per decade, which is attributed to the combination of Volmer-Tafel and Volmer-Heyrovsky mechanism.

An alternative route is using metallic $2 \mathrm{D} 1 \mathrm{~T}-\mathrm{MoS}_{2}$ prepared by the restacking process, and its Tafel slope is 40 to $43 \mathrm{mV}$ per decade. ${ }^{103,104,196}$ As shown in Fig. 8f, edge oxidation study shows that metallic $1 \mathrm{~T}-\mathrm{MoS}_{2}$ provides the whole basal surface as HER catalytic surface, while $2 \mathrm{H}-\mathrm{MoS}_{2}$ shows significantly low catalytic efficiency after edge oxidation. Lithium intercalation of vertically aligned $\mathrm{MoS}_{2}$ film ${ }^{\mathbf{1 1 8}}$ further enhances the catalytic effect, because it results in a decrease of the Mo oxidation state, a modification of the electronic structure by creating a larger interlayer distance, and an enhancement of conductivity by the formation of the $1 \mathrm{~T}$ phase. ${ }^{197}$

\subsection{Photochemistry: catalyst for water splitting and chemical reaction}

In its application as HER catalyst, both types of $2 \mathrm{D} \mathrm{MoS}_{2}$ act as an electrode, which accepts an external electric power source. In contrast, photochemical water splitting is the electrolysis of a water molecule into hydrogen and oxygen gas using (sun-)light, without any electrical power sources. While bulk $\mathrm{MoS}_{2}$ has negligible photochemical water splitting power, ${ }^{198}$ single-layer $2 \mathrm{H}-\mathrm{MoS}_{2}$ has considerable power to split water molecules because single-layer $\mathrm{MoS}_{2}$ has a suitable band structure for water splitting. ${ }^{199}$ For example, the CBM lies $0.41 \mathrm{~V}$ more negative than the proton reduction potential $\left(\mathrm{H}_{2} / \mathrm{H}^{+}, \mathrm{NHE}\right)$, and the VBM is located $0.07 \mathrm{~V}$ more positive than the oxygen reduction potential $\left(\mathrm{O}_{2} / \mathrm{H}_{2} \mathrm{O}, 1.229 \mathrm{~V}\right.$ vs. NHE). The direct bandgap of $1.90 \mathrm{eV}$ is also suitable for accepting sunlight as a power source for water splitting. In an experiment, a photoelectrochemical cell was successfully comprised of an electrode coated with single-layer $\mathrm{MoS}_{2}$, and with $\mathrm{Au}$ nanoparticles further enhancing the efficiency with the aid of surface plasmon. ${ }^{200}$

Another example of $2 \mathrm{D} 2 \mathrm{H}-\mathrm{MoS}_{2}$ as photocatalyst is the photochemical reaction, which accepts external light to excite electrons from the valence band to the conduction band, and then to the target molecules. ${ }^{201}$ As a proof of concept, a dispersion of chemically exfoliated 2D $\mathrm{MoS}_{2}$ flakes is mixed with methylene blue molecule. While pristine methylene blue retains its colour and absorbance for several hours, $20 \mathrm{ppm}$ of $2 \mathrm{D} \mathrm{MoS}_{2}$ results in the photodegradation of methylene blue by $90 \%$ within 2 hours.

\subsection{Biochemistry: various bioapplications of $2 \mathrm{D} \mathrm{MoS}_{2}$ dispersion}

The aqueous dispersion of $2 \mathrm{D} \mathrm{MoS}_{2}$ prepared by the restacking process enables the utilisation of this attractive nanomaterial in the field of biochemistry. In principle, an aqueous dispersion of $2 \mathrm{D} \mathrm{MoS}_{2}$ shows low cytotoxicity and high biocompatibility. ${ }^{202-205}$ Therefore, its adaptation into the human body will enable its application as a multipurpose biomaterial that takes advantage of its remarkable physical and chemical properties. One of the direct bio-applications is antibacterial activity, as confirmed by an experiment on E. coli DH5 $\alpha$ cells (Fig. 9a). ${ }^{202}$ The key idea here is using metallic 2D $1 \mathrm{~T}-\mathrm{MoS}_{2}$ dispersion, where its large
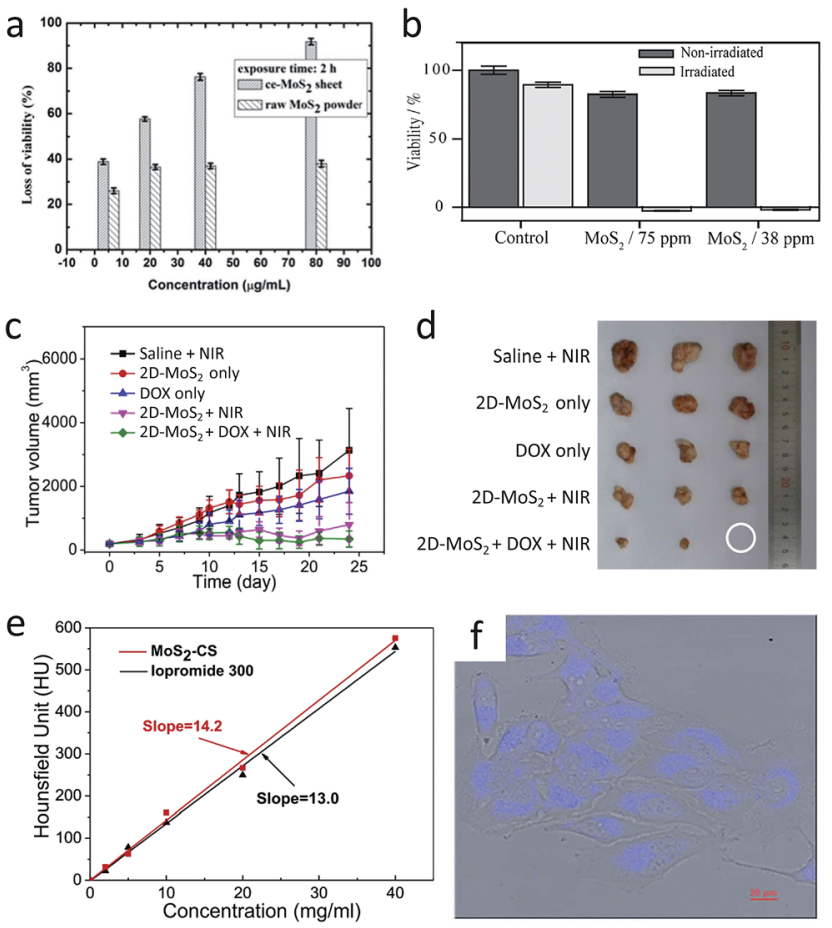

Fig. 9 (a) The change of the viability of $E$. coli $\mathrm{DH} 5 \alpha$ upon exposure to 2D $\mathrm{MoS}_{2}$ suspension upon varying concentration for 2 hours. ${ }^{202}$ Chemically exfoliated samples show higher antibacterial activity than raw ones. (b) The change of viability of HeLa cells with or without treatment with 2D MoS 2 or NIR irradiation. ${ }^{203}$ Most of cells are dead when 2D MoS 2 is injected with NIR irradiation, otherwise most of them are alive. (c) and (d): In vivo phototherapy of pancreatic tumour showing synergetic effect from combination of $2 \mathrm{D} \mathrm{MoS}_{2}$ hyperthermia and doxocurbin chemotherapy, with the daily tumour growth curve (c) and the photographs of the tumour after 24 days of treatment (d). ${ }^{204}$ Here 2D MoS 2 dose is $2.0 \mathrm{mg} \mathrm{kg}^{-1}$, and that of DOX is $0.95 \mathrm{mg} \mathrm{kg}^{-1}$, with NIR (808 nm laser) irradiation of $0.9 \mathrm{~W} \mathrm{~cm}^{-2}$ for 7 minutes. (e) Hounsfield units, the CT contrast value, of different concentrations of the contrasting reagents: $\mathrm{MoS}_{2}$ (red) and commercially used lopromide 300 (black). ${ }^{204}$ (f) A combined image of the photoluminescence image (the purple coloured image) and the optical image (the background and the greyscale image) of HeLa cells, treated with of $1 \mu \mathrm{g} \mathrm{mL}^{-1}$ of $\mathrm{MoS}_{2}{ }^{205}$ Reproduced with permission from: (a) ref. 202 () 2014 Royal Society of Chemistry; (b) ref. 203 @ 2012 John Wiley and Sons; (c), (d) and (e): ref. 204 (c) 2014 American Chemical Society; (f) ref. 205 @ 2014 Royal Society of Chemistry.

surface area and high conductivity easily transfers electrons from the inside of the bacteria cell to its external environment. In addition, 2D $\mathrm{MoS}_{2}$ dispersion generates reactive oxygen species (ROS), which is not observed from graphene oxide (GO).

Another application is photothermal therapy, which takes advantage of its good dispersibility and superior absorbance of near infrared (NIR). ${ }^{203}$ A 2D $\mathrm{MoS}_{2}$ dispersion shows 7.8 times higher absorbance than GO, and its extinction coefficients at $800 \mathrm{~nm}$ is $29.2 \mathrm{~L} \mathrm{~g}^{-1} \mathrm{~cm}^{-1}$, which is higher than gold nanorods. $2 \mathrm{D} \mathrm{MoS}_{2}$ is biocompatible, as confirmed by complexation with $\alpha$-chymotrypsin, where denaturation does not occur. A NIR and 2D $\mathrm{MoS}_{2}$ treatment of HeLa cells shows its superior in vitro photothermal therapy efficiency (Fig. 9b). ${ }^{203}$ In addition, incorporation of chitosan during exfoliation further enhances $\mathrm{MoS}_{2}$ 
biocompatibility and increases the photothermal conversion efficiency up to $24.37 \% .{ }^{204}$ In vivo experiment on mice with pancreatic cancer (Panc-1 tumour) also confirms that chitosanincorporated 2D $\mathrm{MoS}_{2}$ dispersion shows photothermal therapy upon NIR irradiation (Fig. 9c and d). A synergetic effect is created by further incorporation of doxocurbin (DOX), showing remarkable cancer-killing ability, as a result of the combination of hyperthermia and chemotherapy.

Visualisation of certain organs or cells is one of the most important functions for diagnosis and treatment. Based on the large atomic number of Mo, 2D $\mathrm{MoS}_{2}$ flakes can be used as CT image contrast agent. ${ }^{204}$ Its contrasting efficiency is comparable to commercially used Iopromide 300 contrast agent (Fig. 9e). It is also possible to directly visualise cells by PL from $2 \mathrm{D} \mathrm{MoS}_{2}$, as confirmed by the uptake by HeLa cell. ${ }^{205}$ As shown in Fig. 9f, its fluorescence under UV light is observed within live HeLa cells, stating that $2 \mathrm{D} \mathrm{MoS}$ does not harm the cells while retaining its properties.

\section{Conclusions and outlooks}

Atomic layers of $\mathrm{MoS}_{2}$ are one of the most attractive nanomaterials in recent years. Historically, this material has been a topic of research mainly for tribologists, intercalation chemists and petroleum chemists because of its aptness for such applications in the industry. Now, recent surging interests have expanded to scientists and engineers of all fields because of the attractive, useful properties of $2 \mathrm{D} \mathrm{MoS}_{2}$ as an emerging nanomaterial. To this end, substantial research has been carried out in many fields of science and technology, which encompass not only historical research fields but also novel areas. These include nanoelectronics, which mainly concentrates on 2D $\mathrm{MoS}_{2}$ as a direct-gap semiconducting alternative of the metallic graphene. In other fields of research, numerous demonstrations such as FETs, molecular sensors, HER catalysts, or bioapplications have successfully demonstrated its bright potential for future applications. Reflecting upon these important aspects, it is clear that $2 \mathrm{D} \mathrm{MoS}_{2}$ is now much closer to real applications than any other $2 \mathrm{D}$ nanomaterials.

However, there are still many hurdles to overcome in order to bring its full potential into reality. For example, large-scale synthesis of single layers with single crystalline domain is still not achieved. Continuous films of 2D 1T-MoS 2 have not been prepared yet. These high-quality large-scale films are the necessary starting point for applications such as large-scale device fabrication. In addition, catalyst efficiency is still too low to be applied in real industries, and thus further understanding and improvement are required. Heterostructure with other nanomaterials is still an unexplored area of research, which hold the potential of unprecedented, unique nature and applications.

$2 \mathrm{D} \mathrm{MoS}_{2}$ is one of the first 2D nanomaterials that have been studied other than graphene; several experimental techniques, strategic approaches, and demonstration of applications that are applied for graphene have been employed to 2D $\mathrm{MoS}_{2}$ as well. Such comparative studies suggest that these approaches are not only applicable to graphene but also applicable to other two-dimensional nanomaterials, providing a generalised route to study other unexplored $2 \mathrm{D}$ nanomaterials. In this sense, study on $2 \mathrm{D} \mathrm{MoS}$ possesses great importance as a bridge that provides ways to expanding the scope of $2 \mathrm{D}$ nanomaterials.

\section{Acknowledgements}

I. Song, C. Park, and H. C. Choi appreciate the valuable comments by Ms. Lola Brown and Prof. Jiwoong Park at Cornell University. This work was supported by IBS-R014-G2 and NRF2013K1A3A1A32035430. I. Song is grateful to Global Ph.D. Fellowship grant from NRF (NRF-2012H1A2A1016525).

\section{Notes and references}

1 J. G. Hooley, in Preparation and Crystal Growth of Materials with Layered Structures, ed. R. M. A. Lieth, D. Reidel Publishing Company, Dordrecht, 1977, pp. 1-33.

2 A. A. Balchin, in Crystallography and Crystal Chemistry of Materials with Layered Structures, ed. F. Lévy, D. Reidel Publishing Company, Dordrecht, 1976, pp. 1-50.

3 C. Y. Fong and M. Schluter, in Electrons and Phonons in Layered Crystal Structures, ed. T. J. Wieting and M. Schluter, D. Reidel Publishing Company, Dordrecht, 1979, pp. 145-320.

4 R. C. Fivaz and P. E. Schmid, in Optical and Electrical Properties, ed. P. A. Lee, D. Reidel Publishing Company, Dordrecht, 1976, pp. 343-384.

5 Preparation and Crystal Growth of Materials with Layered Structures, ed. R. M. A. Lieth, D. Reidel Publishing Company, Dordrecht, 1977.

6 M. M. Thackeray, J. O. Thomas and M. S. Whittingham, MRS Bull., 2000, 25, 39-46.

7 M. S. Dresselhaus, in Intercalation in Layered Materials, ed. M. S. Dresselhaus, Plenum Press, New York, 1986, pp. 1-30.

8 T. Bartels, W. Bock, J. Braun, C. Busch, W. Buss, W. Dresel, C. Freiler, M. Harperscheid, R.-P. Heckler, D. Hörner, F. Kubicki, G. Lingg, A. Losch, R. Luther, T. Mang, S. Noll and J. Omeis, in Ullmann's Encyclopedia of Industrial Chemistry, John Wiley and Sons-VCH Verlag GmbH \& Co. KGaA, 2000.

9 K. S. Novoselov, A. K. Geim, S. V. Morozov, D. Jiang, Y. Zhang, S. V. Dubonos, I. V. Grigorieva and A. A. Firsov, Science, 2004, 306, 666-669.

10 K. S. Novoselov, D. Jiang, F. Schedin, T. J. Booth, V. V. Khotkevich, S. V. Morozov and A. K. Geim, Proc. Natl. Acad. Sci. U. S. A., 2005, 102, 10451-10453.

11 N. Coleman, M. Lotya, A. O'Neill, S. D. Bergin, P. J. King, U. Khan, K. Young, A. Gaucher, S. De, R. J. Smith, I. V. Shvets, S. K. Arora, G. Stanton, H. Y. Kim, K. Lee, G. T. Kim, G. S. Duesberg, T. Hallam, J. J. Boland, J. J. Wang, J. F. Donegan, J. C. Grunlan, G. Moriarty, A. Shmeliov, R. J. Nicholls, J. M. Perkins, E. M. Grieveson, K. Theuwissen, D. W. McComb, P. D. Nellist and V. Nicolosi, Science, 2011, 331, 568-571.

12 A. K. Geim and K. S. Novoselov, Nat. Mater., 2007, 6, 183191. 
13 Q. H. Wang, K. Kalantar-Zadeh, A. Kis, J. N. Coleman and M. S. Strano, Nat. Nanotechnol., 2012, 7, 699-712.

14 M. Chhowalla, H. S. Shin, G. Eda, L.-J. Li, K. P. Loh and H. Zhang, Nat. Chem., 2013, 5, 263-275.

15 G. A. Tsigdinos, in Aspects of Molybdenum and Related Chemistry, Springer-Verlag Berlin Heidelberg, New York, 1978, vol. 76, pp. 65-105.

16 R. F. Sebenik, A. R. Burkin, R. R. Dorfler, J. M. Laferty, G. Leichtfried, H. Meyer-Grünow, P. C. H. Mitchell, M. S. Vukasovich, D. A. Church, G. G. Van Riper, J. C. Gilliland and S. A. Thielke, Molybdenum and Molybdenum Compounds, Wiley-VCH Verlag GmbH \& Co. KGaA, 2000.

17 E. R. Braithwaite and J. Haber, Molybdenum: an outline of its chemistry and uses, Elsevier, 1994.

18 W. Koehler, US Pat., 1714 564, 1929.

19 H. G. M. Fischer and R. P. Russell, US Pat., 2322 622, 1943.

20 R. P. Haering, J. A. R. Stiles and K. Brandt, US Pat., 4224 390, 1980.

21 Y. Feldman, E. Wasserman, D. J. Srolovitz and R. Tenne, Science, 1995, 267, 222-225.

22 Y. Feldman, G. L. Frey, M. Homyonfer, V. Lyakhovitskaya, L. Margulis, H. Cohen, G. Hodes, J. L. Hutchison and R. Tenne, J. Am. Chem. Soc., 1996, 118, 5362-5367.

23 G. L. Frey, R. Tenne, M. J. Matthews, M. S. Dresselhaus and G. Dresselhaus, J. Mater. Res., 1998, 13, 2412-2417.

24 R. Tenne, in The Chemistry of Nanostructured Materials, ed. P. Yang, World Sci. Publ., Singapore, 2003, pp. 147-182.

25 K. F. Mak, C. Lee, J. Hone, J. Shan and T. F. Heinz, Phys. Rev. Lett., 2010, 105, 136805.

26 B. Radisavljevic, A. Radenovic, J. Brivio, V. Giacometti and A. Kis, Nat. Nanotechnol., 2011, 6, 147-150.

27 K. F. Mak, K. He, J. Shan and T. F. Heinz, Nat. Nanotechnol., 2012, 7, 494-498.

28 H. Zeng, J. Dai, W. Yao, D. Xiao and X. Cui, Nat. Nanotechnol., 2012, 7, 490-493.

29 T. Cao, G. Wang, W. Han, H. Ye, C. Zhu, J. Shi, Q. Niu, P. Tan, E. Wang, B. Liu and J. Feng, Nat. Commun., 2012, 3, 887.

30 S. Wu, C. Huang, G. Aivazian, J. S. Ross, D. H. Cobden and X. Xu, ACS Nano, 2013, 7, 2768-2772.

31 T. F. Jaramillo, K. P. Jørgensen, J. Bonde, J. H. Nielsen, S. Horch and I. Chorkendorff, Science, 2007, 317, 100-102.

32 B. Hinnemann, P. G. Moses, J. Bonde, K. P. Jørgensen, J. H. Nielsen, S. Horch, I. Chorkendorff and J. K. Nørskov, J. Am. Chem. Soc., 2005, 127, 5308-5309.

33 H. Li, Z. Yin, Q. He, H. Li, X. Huang, G. Lu, D. W. H. Fam, A. I. Y. Tok, Q. Zhang and H. Zhang, Small, 2012, 8, 63-67.

34 F. E. Wickman and D. K. Smith, Am. Mineral., 1970, 55, 1843-1856.

35 R. G. Dickinson and L. Pauling, J. Am. Chem. Soc., 1923, 45, 1466-1471.

36 F. Jellinek, G. Brauer and H. Muller, Nature, 1960, 185, 376377.

37 F. Wypych and R. Schollhorn, J. Chem. Soc., Chem. Commun., 1992, 1386-1388.
38 A. N. Enyashin, L. Yadgarov, L. Houben, I. Popov, M. Weidenbach, R. Tenne, M. Bar-Sadan and G. Seifert, J. Phys. Chem. C, 2011, 115, 24586-24591.

39 F. Wypych, T. Weber and R. Prins, Chem. Mater., 1998, 10, 723-727.

40 R. V. Kasowski, Phys. Rev. Lett., 1973, 30, 1175-1178.

41 R. Fivaz and E. Mooser, Phys. Rev., 1967, 163, 743-755.

42 A. N. Enyashin and G. Seifert, Comput. Theor. Chem., 2012, 999, 13-20.

43 R. Huisman, R. de Jonge, C. Haas and F. Jellinek, J. Solid State Chem., 1971, 3, 56-66.

44 D. W. Bullett, J. Phys. C: Solid State Phys., 1978, 11, 45014514.

45 W. Jaegermann and H. Tributsch, Prog. Surf. Sci., 1988, 29, 1-167.

46 V. Alexiev, R. Prins and T. Weber, Phys. Chem. Chem. Phys., 2000, 2, 1815-1827.

47 A. Clark and R. H. Williams, J. Phys. D: Appl. Phys., 1968, 1, 1222-1224.

48 R. Suzuki, M. Sakano, Y. J. Zhang, R. Akashi, D. Morikawa, a. Harasawa, K. Yaji, K. Kuroda, K. Miyamoto, T. Okuda, K. Ishizaka, R. Arita and Y. Iwasa, Nat. Nanotechnol., 2014, 9, 611-617.

49 T. Wieting and J. Verble, Phys. Rev. B: Condens. Matter Mater. Phys., 1971, 3, 4286-4292.

50 J. L. Verble, T. J. Wietling and P. R. Reed, Solid State Commun., 1972, 11, 941-944.

51 W. Y. Liang, in Intercalation in Layered Materials, ed. M. S. Dresselhaus, Plenum Press, New York, 1986, pp. 31-73.

52 Z.-H. Chi, X.-M. Zhao, H. Zhang, A. F. Goncharov, S. S. Lobanov, T. Kagayama, M. Sakata and X.-J. Chen, Phys. Rev. Lett., 2014, 113, 036802.

53 J. A. Woollam and R. B. Somoano, Phys. Rev. B: Condens. Matter Mater. Phys., 1976, 13, 3843-3853.

54 R. Somoano and A. Rembaum, Phys. Rev. Lett., 1971, 27, 402-404.

55 D. Liu, X. Chen, D. Li, F. Wang, X. Luo and B. Yang, J. Mol. Struct., 2010, 980, 66-71.

56 D. Godfrey and E. C. Nelson, Oxidation Characteristic of Molybdenum Disulfide and Effect of Such Oxidation on Its Role as a Solid-film Lubricant: N.A.C.A. Technical Note No. 1882, National Advisory Committee for Aeronautics, Washington, D. C., 1949.

57 J. A. Wilson and A. D. Yoffe, Adv. Phys., 1969, 18, 193-335.

58 Q. Li, E. C. Walter, W. E. van der Veer, B. J. Murray, J. T. Newberg, E. W. Bohannan, J. A. Switzer, J. C. Hemminger and R. M. Penner, J. Phys. Chem. B, 2005, 109, 3169-3182.

59 J. W. Frondel and F. E. Wickman, Am. Mineral., 1970, 55, 1857-1875.

60 J. R. Stubbles and F. D. Richardson, Trans. Faraday Soc., 1960, 56, 1460-1466.

61 T. Prasad, J. Inorg. Nucl. Chem., 1973, 35, 1895-1904.

62 R. E. Bell and R. E. Herfert, J. Am. Chem. Soc., 1957, 79, 3351-3354.

63 M. S. Silverman, Inorg. Chem., 1967, 6, 1063-1064. 
64 J. C. Wildervanck and F. Jellinek, Z. Anorg. Allg. Chem., 1964, 328, 309-318.

65 W. O. Winer, Wear, 1967, 10, 422-452.

66 R. Holinski and J. Gänsheimer, Wear, 1972, 19, 329-342.

67 P. D. Fleischauer, J. R. Lince, P. A. Bertrand and R. Bauer, Langmuir, 1989, 5, 1009-1015.

68 A. R. Lansdown, Molybdenum Disulphide Lubrication, Elsevier Science, Amsterdam, 1999.

69 J. M. Martin, H. Pascal, C. Donnet, T. Le Mogne, J. L. Loubet and T. Epicier, Surf. Coat. Technol., 1994, 68-69, 427-432.

70 D. F. Moore, Principles and Applications of Tribology, Pergamon Press Inc., New York, 1975.

71 A. Savan, E. Pflüger, P. Voumard, A. Schröer and M. Simmonds, Lubr. Sci., 2000, 12, 185-203.

72 R. H. Savage, J. Appl. Phys., 1948, 19, 1-10.

73 P. J. Bryant, P. L. Gutshall and L. H. Taylor, Wear, 1964, 7, 118-126.

74 A. J. Haltner, Wear, 1964, 7, 102-117.

75 A. J. Haltner and C. S. Oliver, Ind. Eng. Chem. Fundam., 1966, 5, 348-355.

76 L. W. Vernon and J. T. Richardson, US Pat., 3000 816, 1961.

77 H. Topsøe, B. S. Clausen and F. E. Massoth, in CatalysisScience and Technology, ed. J. R. Anderson and M. Boudart, Springer Berlin Heidelberg, Berlin, Heidelberg, 1996, pp. 1-269.

78 L. S. Byskov, J. K. Nørskov, B. S. Clausen and H. Topsøe, J. Catal., 1999, 187, 109-122.

79 F. Wypych, K. Sollmann and R. Schöllhorn, Mater. Res. Bull., 1992, 27, 545-553.

80 Y.-C. Lin, D. O. Dumcenco, Y.-S. Huang and K. Suenaga, Nat. Nanotechnol., 2014, 9, 391-396.

81 P. J. Mulhern, Can. J. Phys., 1989, 67, 1049-1052.

82 D. Yang, S. Sandoval, W. Divigalpitiya, J. Irwin and R. Frindt, Phys. Rev. B: Condens. Matter Mater. Phys., 1991, 43, 12053-12056.

83 K. E. Dungey, M. D. Curtis and J. E. Penner-Hahn, Chem. Mater., 1998, 10, 2152-2161.

84 J. Heising and M. G. Kanatzidis, J. Am. Chem. Soc., 1999, 121, 11720-11732.

85 E. Benavente, M. A. S. Ana, F. Mendizábal and G. González, Coord. Chem. Rev., 2002, 224, 87-109.

86 C. Julien, S. I. Saikh and G. A. Nazri, Mater. Sci. Eng. B, 1992, 15, 73-77.

87 M. B. Dines, Science, 1975, 188, 1210-1211.

88 R. Bissessur, J. Heising, W. Hirpo and M. Kanatzidis, Chem. Mater., 1996, 8, 318-320.

89 L. Wang, J. Schindler, J. A. Thomas, C. R. Kannewurf and M. G. Kanatzidis, Chem. Mater., 1995, 7, 1753-1755.

90 T. Stephenson, Z. Li, B. Olsen and D. Mitlin, Energy Environ. Sci., 2014, 7, 209-231.

91 J. O. Besenhard, J. Yang and M. Winter, J. Power Sources, 1997, 68, 87-90.

92 H. Hwang, H. Kim and J. Cho, Nano Lett., 2011, 11, 4826-4830.

93 Y. Y. Wang, R. X. Gao, Z. H. Ni, H. He, S. P. Guo, H. P. Yang, C. X. Cong and T. Yu, Nanotechnology, 2012, 23, 495713.

94 M. M. Benameur, B. Radisavljevic, J. S. Héron, S. Sahoo, H. Berger and A. Kis, Nanotechnology, 2011, 22, 125706.
95 P. Blake, E. W. Hill, A. H. Castro Neto, K. S. Novoselov, D. Jiang, R. Yang, T. J. Booth and A. K. Geim, Appl. Phys. Lett., 2007, 91, 063124.

96 H. Li, G. Lu, Z. Yin, Q. He, H. Li, Q. Zhang and H. Zhang, Small, 2012, 8, 682-686.

97 A. Castellanos-Gomez, M. Barkelid, A. M. Goossens, V. E. Calado, H. S. J. van der Zant and G. A. Steele, Nano Lett., 2012, 12, 3187-3192.

98 R. Ruoff, Nat. Nanotechnol., 2008, 3, 10-11.

99 P. Joensen, R. F. Frindt and S. R. Morrison, Mater. Res. Bull., 1986, 21, 457-461.

100 H. S. S. Ramakrishna Matte, A. Gomathi, A. K. Manna, D. J. Late, R. Datta, S. K. Pati and C. N. R. Rao, Angew. Chem., Int. Ed., 2010, 49, 4059-4062.

101 Z. Zeng, Z. Yin, X. Huang, H. Li, Q. He, G. Lu, F. Boey and H. Zhang, Angew. Chem., Int. Ed., 2011, 50, 11093-11097.

102 G. Eda, T. Fujita, H. Yamaguchi, D. Voiry, M. Chen and M. Chhowalla, ACS Nano, 2012, 6, 7311-7317.

103 M. A. Lukowski, A. S. Daniel, F. Meng, A. Forticaux, L. Li and S. Jin, J. Am. Chem. Soc., 2013, 135, 10274-10277.

104 D. Voiry, M. Salehi, R. Silva, T. Fujita, M. Chen, T. Asefa, V. B. Shenoy, G. Eda and M. Chhowalla, Nano Lett., 2013, 13, 6222-6227.

105 R. J. Smith, P. J. King, M. Lotya, C. Wirtz, U. Khan, S. De, A. O'Neill, G. S. Duesberg, J. C. Grunlan, G. Moriarty, J. Chen, J. Wang, A. I. Minett, V. Nicolosi and J. N. Coleman, Adv. Mater., 2011, 23, 3944-3948.

106 P. Afanasiev, G.-F. Xia, G. Berhault, B. Jouguet and M. Lacroix, Chem. Mater., 1999, 11, 3216-3219.

107 K.-G. Zhou, N.-N. Mao, H.-X. Wang, Y. Peng and H.-L. Zhang, Angew. Chem., Int. Ed., 2011, 50, 10839-10842.

108 G. Cunningham, M. Lotya, C. S. Cucinotta, S. Sanvito, S. D. Bergin, R. Menzel, M. S. P. Shaffer and J. N. Coleman, ACS Nano, 2012, 6, 3468-3480.

109 N. Liu, P. Kim, J. H. Kim, J. H. Ye, S. Kim and C. J. Lee, ACS Nano, 2014, 8, 6902-6910.

110 K. Lee, H.-Y. Kim, M. Lotya, J. N. Coleman, G.-T. Kim and G. S. Duesberg, Adv. Mater., 2011, 23, 4178-4182.

111 G. Eda, H. Yamaguchi, D. Voiry, T. Fujita, M. Chen and M. Chhowalla, Nano Lett., 2011, 11, 5111-5116.

112 A. O'Neill, U. Khan and J. N. Coleman, Chem. Mater., 2012, 24, 2414-2421.

113 J. Brivio, D. T. L. Alexander and A. Kis, Nano Lett., 2011, 11, 5148-5153.

114 S. Helveg, J. Lauritsen, E. Laegsgaard, I. Stensgaard, J. Nørskov, B. Clausen, H. Tøpsoe and F. Besenbacher, Phys. Rev. Lett., 2000, 84, 951-954.

115 D. Kim, D. Sun, W. Lu, Z. Cheng, Y. Zhu, D. Le, T. S. Rahman and L. Bartels, Langmuir, 2011, 27, 1165011653.

116 Y. Zhan, Z. Liu, S. Najmaei, P. M. Ajayan and J. Lou, Small, 2012, 8, 966-971.

117 Y. Lee, J. Lee, H. Bark, I.-K. Oh, G. H. Ryu, Z. Lee, H. Kim, J. H. Cho, J.-H. Ahn and C. Lee, Nanoscale, 2014, 6, 28212826.

118 D. Kong, H. Wang, J. J. Cha, M. Pasta, K. J. Koski, J. Yao and Y. Cui, Nano Lett., 2013, 13, 1341-1347. 
119 M. Schleberger, D. Fujita, C. Scharfschwerdt and S. Tougaard, Surf. Sci., 1995, 331-333, 942-947.

120 Y. Shi, W. Zhou, A.-Y. Lu, W. Fang, Y.-H. Lee, A. L. Hsu, S. M. Kim, K. K. Kim, H. Y. Yang, L.-J. Li, J.-C. Idrobo and J. Kong, Nano Lett., 2012, 12, 2784-2791.

121 K.-K. Liu, W. Zhang, Y.-H. Lee, Y.-C. Lin, M.-T. Chang, C.-Y. Su, C.-S. Chang, H. Li, Y. Shi, H. Zhang, C.-S. Lai and L.-J. Li, Nano Lett., 2012, 12, 1538-1544.

122 Y.-H. Lee, X.-Q. Zhang, W. Zhang, M.-T. Chang, C.-T. Lin, K.-D. Chang, Y.-C. Yu, J. T.-W. Wang, C.-S. Chang, L.-J. Li and T.-W. Lin, Adv. Mater., 2012, 24, 2320-2325.

123 Y.-C. Lin, W. Zhang, J.-K. Huang, K.-K. Liu, Y.-H. Lee, C.-T. Liang, C.-W. Chu and L.-J. Li, Nanoscale, 2012, 4, 6637-6641.

124 Y. Yu, C. Li, Y. Liu, L. Su, Y. Zhang and L. Cao, Sci. Rep., 2013, 3, 1866.

125 X. Wang, H. Feng, Y. Wu and L. Jiao, J. Am. Chem. Soc., 2013, 135, 5304-5307.

126 Y.-H. Lee, L. Yu, H. Wang, W. Fang, X. Ling, Y. Shi, C.-T. Lin, J.-K. Huang, M.-T. Chang, C.-S. Chang, M. Dresselhaus, T. Palacios, L.-J. Li and J. Kong, Nano Lett., 2013, 13, 1852-1857.

127 Q. Ji, Y. Zhang, T. Gao, Y. Zhang, D. Ma, M. Liu, Y. Chen, X. Qiao, P.-H. Tan, M. Kan, J. Feng, Q. Sun and Z. Liu, Nano Lett., 2013, 13, 3870-3877.

128 I. Song, C. Park, M. Hong, J. Baik, H.-J. Shin and H. C. Choi, Angew. Chem., Int. Ed., 2014, 53, 1266-1269.

129 A. M. van der Zande, P. Y. Huang, D. A. Chenet, T. C. Berkelbach, Y. You, G.-H. Lee, T. F. Heinz, D. R. Reichman, D. A. Muller and J. C. Hone, Nat. Mater., 2013, 12, 554-561.

130 Y. Liu, R. Ghosh, D. Wu, A. Ismach, R. Ruoff and K. Lai, Nano Lett., 2014, 14, 4682-4686.

131 P. Y. Huang, C. S. Ruiz-Vargas, A. M. van der Zande, W. S. Whitney, M. P. Levendorf, J. W. Kevek, S. Garg, J. S. Alden, C. J. Hustedt, Y. Zhu, J. Park, P. L. McEuen and D. A. Muller, Nature, 2011, 469, 389-392.

132 S. Najmaei, Z. Liu, W. Zhou, X. Zou, G. Shi, S. Lei, B. I. Yakobson, J.-C. Idrobo, P. M. Ajayan and J. Lou, Nat. Mater., 2013, 12, 754-759.

133 W. Zhou, X. Zou, S. Najmaei, Z. Liu, Y. Shi, J. Kong, J. Lou, P. M. Ajayan, B. I. Yakobson and J.-C. Idrobo, Nano Lett., 2013, 13, 2615-2622.

134 S. Najmaei, M. Amani, M. L. Chin, Z. Liu, A. G. Birdwell, T. P. O'Regan, P. M. Ajayan, M. Dubey and J. Lou, ACS Nano, 2014, 8, 7930-7937.

135 A. Splendiani, L. Sun, Y. Zhang, T. Li, J. Kim, C.-Y. Chim, G. Galli and F. Wang, Nano Lett., 2010, 10, 12711275.

136 X. Li, X. Wang, L. Zhang, S. Lee and H. Dai, Science, 2008, 319, 1229-1232.

137 T. Böker, R. Severin, A. Müller, C. Janowitz, R. Manzke, D. Voß, P. Krüger, A. Mazur and J. Pollmann, Phys. Rev. B: Condens. Matter Mater. Phys., 2001, 64, 235305.

138 S. Lebègue and O. Eriksson, Phys. Rev. B: Condens. Matter Mater. Phys., 2009, 79, 115409.
139 C. R. Zhu, G. Wang, B. L. Liu, X. Marie, X. F. Qiao, X. Zhang, X. X. Wu, H. Fan, P. H. Tan, T. Amand and B. Urbaszek, Phys. Rev. B: Condens. Matter Mater. Phys., 2013, 88, 121301.

140 H. J. Conley, B. Wang, J. I. Ziegler, R. F. Haglund, S. T. Pantelides and K. I. Bolotin, Nano Lett., 2013, 13, 3626-3630.

141 A. Castellanos-Gomez, R. Roldán, E. Cappelluti, M. Buscema, F. Guinea, H. S. J. van der Zant and G. A. Steele, Nano Lett., 2013, 13, 5361-5366.

142 S. N. Shirodkar and U. V. Waghmare, Phys. Rev. Lett., 2014, 112, 157601.

143 D. Jena and A. Konar, Phys. Rev. Lett., 2007, 98, 136805.

144 M. S. Fuhrer and J. Hone, Nat. Nanotechnol., 2013, 8, 146147.

145 B. Radisavljevic and A. Kis, Nat. Nanotechnol., 2013, 8, 147148.

146 K. Kaasbjerg, K. S. Thygesen and K. W. Jacobsen, Phys. Rev. B: Condens. Matter Mater. Phys., 2012, 85, 115317.

147 S. Kim, A. Konar, W.-S. Hwang, J. H. Lee, J. Lee, J. Yang, C. Jung, H. Kim, J.-B. Yoo, J.-Y. Choi, Y. W. Jin, S. Y. Lee, D. Jena, W. Choi and K. Kim, Nat. Commun., 2012, 3, 1011.

148 F. L. Deepak, H. Cohen, S. Cohen, Y. Feldman, R. PopovitzBiro, D. Azulay, O. Millo and R. Tenne, J. Am. Chem. Soc., 2007, 129, 12549-12562.

149 V. V. Ivanovskaya, T. Heine, S. Gemming and G. Seifert, Phys. Status Solidi B, 2006, 243, 1757-1764.

150 M. R. Laskar, D. N. Nath, L. Ma, E. W. Lee, C. H. Lee, T. Kent, Z. Yang, R. Mishra, M. A. Roldan, J.-C. Idrobo, S. T. Pantelides, S. J. Pennycook, R. C. Myers, Y. Wu and S. Rajan, Appl. Phys. Lett., 2014, 104, 092104.

151 Y.-C. Lin, D. O. Dumcenco, H.-P. Komsa, Y. Niimi, A. V. Krasheninnikov, Y.-S. Huang and K. Suenaga, Adv. Mater., 2014, 26, 2857-2861.

152 L. Yadgarov, R. Rosentsveig, G. Leitus, A. Albu-Yaron, A. Moshkovich, V. Perfilyev, R. Vasic, A. I. Frenkel, A. N. Enyashin, G. Seifert, L. Rapoport and R. Tenne, Angew. Chem., Int. Ed., 2011, 2, 1-5.

153 K. Dolui, I. Rungger, C. Das Pemmaraju and S. Sanvito, Phys. Rev. B: Condens. Matter Mater. Phys., 2013, 88, 075420.

154 H. Fang, M. Tosun, G. Seol, T. C. Chang, K. Takei, J. Guo and A. Javey, Nano Lett., 2013, 13, 1991-1995.

155 K. Dolui, I. Rungger and S. Sanvito, Phys. Rev. B: Condens. Matter Mater. Phys., 2013, 87, 165402.

156 B. Radisavljevic and A. Kis, Nat. Mater., 2013, 12, 815-820. 157 J. T. Ye, Y. J. Zhang, R. Akashi, M. S. Bahramy, R. Arita and Y. Iwasa, Science, 2012, 338, 1193-1196.

158 K. Taniguchi, A. Matsumoto, H. Shimotani and H. Takagi, Appl. Phys. Lett., 2012, 101, 042603.

159 I. Popov, G. Seifert and D. Tománek, Phys. Rev. Lett., 2012, 108, 156802.

160 S. Das, H.-Y. Chen, A. V. Penumatcha and J. Appenzeller, Nano Lett., 2013, 13, 100-105.

161 J. Kang, W. Liu and K. Banerjee, Appl. Phys. Lett., 2014, 104, 093106.

162 Y. Yoon, K. Ganapathi and S. Salahuddin, Nano Lett., 2011, 11, 3768-3773. 
163 H.-Y. Chang, S. Yang, J. Lee, L. Tao, W.-S. Hwang, D. Jena, N. Lu and D. Akinwande, ACS Nano, 2013, 7, 5446-5452.

164 Y. Zhang, J. Ye, Y. Matsuhashi and Y. Iwasa, Nano Lett., 2012, 12, 1136-1140.

165 W. Bao, X. Cai, D. Kim, K. Sridhara and M. S. Fuhrer, Appl. Phys. Lett., 2013, 102, 042104.

166 Y. J. Zhang, J. T. Ye, Y. Yomogida, T. Takenobu and Y. Iwasa, Nano Lett., 2013, 13, 3023-3028.

167 B. Radisavljevic, M. B. Whitwick and A. Kis, ACS Nano, 2011, 5, 9934-9938.

168 T. Cheiwchanchamnangij and W. R. L. Lambrecht, Phys. Rev. B: Condens. Matter Mater. Phys., 2012, 85, 205302.

169 Y. Wang, J. Z. Ou, S. Balendhran, A. F. Chrimes, M. Mortazavi, D. D. Yao, M. R. Field, K. Latham, V. Bansal, J. R. Friend, S. Zhuiykov, N. V. Medhekar, M. S. Strano and K. Kalantar-zadeh, ACS Nano, 2013, 7, 10083-10093.

170 K. F. Mak, K. L. McGill, J. Park and P. L. McEuen, Science, 2014, 344, 1489-1492.

171 K. F. Mak, K. He, C. Lee, G. H. Lee, J. Hone, T. F. Heinz and J. Shan, Nat. Mater., 2013, 12, 207-211.

172 S. Mouri, Y. Miyauchi and K. Matsuda, Nano Lett., 2013, 13, 5944-5948.

173 C. Lee, H. Yan, L. E. Brus, T. F. Heinz, J. Hone and S. Ryu, ACS Nano, 2010, 4, 2695-2700.

174 A. Molina-Sánchez and L. Wirtz, Phys. Rev. B: Condens. Matter Mater. Phys., 2011, 84, 155413.

175 H. Li, Q. Zhang, C. C. R. Yap, B. K. Tay, T. H. T. Edwin, A. Olivier and D. Baillargeat, Adv. Funct. Mater., 2012, 22, 1385-1390.

176 L. Sun, J. Yan, D. Zhan, L. Liu, H. Hu, H. Li, B. K. Tay, J.-L. Kuo, C.-C. Huang, D. W. Hewak, P. S. Lee and Z. X. Shen, Phys. Rev. Lett., 2013, 111, 126801.

177 M. Buscema, M. Barkelid, V. Zwiller, H. S. J. van der Zant, G. A. Steele and A. Castellanos-Gomez, Nano Lett., 2013, 13, 358-363.

178 Z. Yin, H. Li, H. Li, L. Jiang, Y. Shi, Y. Sun, G. Lu, Q. Zhang, X. Chen and H. Zhang, ACS Nano, 2012, 6, 74-80.

179 O. Lopez-Sanchez, D. Lembke, M. Kayci, A. Radenovic and A. Kis, Nat. Nanotechnol., 2013, 8, 497-501.

180 W. Zhang, J.-K. Huang, C.-H. Chen, Y.-H. Chang, Y.-J. Cheng and L.-J. Li, Adv. Mater., 2013, 25, 3456-3461.

181 H. S. Lee, S.-W. Min, Y.-G. Chang, M. K. Park, T. Nam, H. Kim, J. H. Kim, S. Ryu and S. Im, Nano Lett., 2012, 12, 3695-3700.

182 M.-L. Tsai, S.-H. Su, J.-K. Chang, D.-S. Tsai, C.-H. Chen, C.-I. Wu, L.-J. Li, L.-J. Chen and J.-H. He, ACS Nano, 2014, 8, 8317-8322.

183 X. Gu, W. Cui, H. Li, Z. Wu, Z. Zeng, S.-T. Lee, H. Zhang and B. Sun, Adv. Energy Mater., 2013, 3, 1262-1268.
184 R. S. Sundaram, M. Engel, A. Lombardo, R. Krupke, A. C. Ferrari, P. Avouris and M. Steiner, Nano Lett., 2013, 13, 1416-1421.

185 S. Bertolazzi, J. Brivio and A. Kis, ACS Nano, 2011, 5, 97039709.

186 A. Castellanos-Gomez, M. Poot, G. a. Steele, H. S. J. van der Zant, N. Agraït and G. Rubio-Bollinger, Adv. Mater., 2012, 24, 772-775.

187 D. Sarkar, W. Liu, X. Xie, A. C. Anselmo, S. Mitragotri and K. Banerjee, ACS Nano, 2014, 8, 3992-4003.

188 D. J. Late, Y. Huang, B. Liu, J. Acharya, S. N. Shirodkar, J. Luo, A. Yan, D. Charles, U. V Waghmare, V. P. Dravid and C. N. R. Rao, ACS Nano, 2013, 7, 4879-4891.

189 Q. Yue, Z. Shao, S. Chang and J. Li, Nanoscale Res. Lett., 2013, 8, 425.

190 C. Zhu, Z. Zeng, H. Li, F. Li, C. Fan and H. Zhang, J. Am. Chem. Soc., 2013, 135, 5998-6001.

191 A. Tuxen, J. Kibsgaard, H. Gøbel, E. Lægsgaard, H. Topsøe, J. V. Lauritsen and F. Besenbacher, ACS Nano, 2010, 4, 4677-4682.

192 R. R. Chianelli, M. H. Siadati, M. P. De la Rosa, G. Berhault, J. P. Wilcoxon, R. Bearden and B. L. Abrams, Catal. Rev., 2006, 48, 1-41.

193 A. Sobczynski, J. Catal., 1991, 131, 156-166.

194 Y. Li, H. Wang, L. Xie, Y. Liang, G. Hong and H. Dai, J. Am. Chem. Soc., 2011, 133, 7296-7299.

195 X. Huang, Z. Zeng, S. Bao, M. Wang, X. Qi, Z. Fan and H. Zhang, Nat. Commun., 2013, 4, 1444.

196 U. Maitra, U. Gupta, M. De, R. Datta, A. Govindaraj and C. N. R. Rao, Angew. Chem., Int. Ed., 2013, 52, 13057-13061.

197 H. Wang, Z. Lu, S. Xu, D. Kong, J. J. Cha, G. Zheng, P.-C. Hsu, K. Yan, D. Bradshaw, F. B. Prinz and Y. Cui, Proc. Natl. Acad. Sci. U. S. A., 2013, 110, 19701-19706.

198 X. Zong, H. Yan, G. Wu, G. Ma, F. Wen, L. Wang and C. Li, J. Am. Chem. Soc., 2008, 130, 7176-7177.

199 Y. Li, Y.-L. Li, C. M. Araujo, W. Luo and R. Ahuja, Catal. Sci. Technol., 2013, 3, 2214-2220.

200 Z. Yin, B. Chen, M. Bosman, X. Cao, J. Chen, B. Zheng and H. Zhang, Small, 2014, 10, 3537-3543.

201 M. D. J. Quinn, N. H. Ho and S. M. Notley, ACS Appl. Mater. Interfaces, 2013, 5, 12751-12756.

202 X. Yang, J. Li, T. Liang, C. Ma, Y. Zhang, H. Chen, N. Hanagata, H. Su and M. Xu, Nanoscale, 2014, 6, 1012610133.

203 S. S. Chou, B. Kaehr, J. Kim, B. M. Foley, M. De, P. E. Hopkins, J. Huang, C. J. Brinker and V. P. Dravid, Angew. Chem., Int. Ed., 2013, 52, 4160-4164.

204 W. Yin, L. Yan, J. Yu, G. Tian, L. Zhou, X. Zheng, X. Zhang, Y. Yong, J. Li, Z. Gu and Y. Zhao, ACS Nano, 2014, 8, 69226933.

205 R. Anbazhagan, H.-J. Wang, H.-C. Tsai and R.-J. Jeng, RSC Adv., 2014, 4, 42936-42941. 